\title{
AN INVITATION TO KÄHLER-EINSTEIN METRICS AND RANDOM POINT PROCESSES
}

\author{
ROBERT J. BERMAN \\ Dedicated to Shing-Tung Yau on the occasion of his 70th birthday.
}

\begin{abstract}
This is an invitation to the probabilistic approach for constructing KählerEinstein metrics on complex projective algebraic manifolds $X$. The metrics in question emerge in the large $N$-limit from a canonical way of sampling $N$ points on $X$, i.e. from random point processes on $X$, defined in terms of algebro-geometric data. The proof of the convergence towards Kähler-Einstein metrics with negative Ricci curvature is explained. In the case of positive Ricci curvature a variational approach is introduced to prove the conjectural convergence, which can be viewed as a probabilistic constructive analog of the Yau-Tian-Donaldson conjecture. The variational approach reveals, in particular, that the convergence holds under the hypothesis that there is no phase transition, which - from the algebro-geometric point of view - amounts to an analytic property of a certain Archimedean zeta function.
\end{abstract}

\section{INTRODUCTION}

A central theme in current complex geometry is the interplay between the differential geometry of Kähler-Einstein metrics on a compact complex manifold $X$ and complex algebraic geometry. These connections were emphasized by Yau in the 80s [82, leading up to the formulation of the Yau-Tian-Donaldson conjecture involving the notion of K-stability [76, 42. This paper is as an invitation to the probabilistic approach to Kähler-Einstein metrics, initiated in [10, 11, 8]. From this probabilistic point of view the Kähler-Einstein metrics emerge in the large $N$-limit from a canonical way of sampling $N$ points on $X$, i.e. from canonical random point process on $X$. The point processes are defined in terms of algebro-geometric data and furnish canonical and explicit approximations to Kähler-Einstein metrics, expressed as period integrals. The thrust of this approach is thus that it provides a new explicit bridge between Kähler-Einstein metrics and algebraic geometry. Moreover, it leads to a new notion of stability, dubbed Gibbs stability, which naturally fits into the logarithmic setup of the Minimal Model Progrom of current birational algebraic geometry. The investigation of the large $N$-limit of the point processes also naturally ties in with the variational approach to Kähler-Einstein metrics in [18, 19, 20] (via the notion a large devition principle).

The aim of the present work is to provide both a survey of the probabilistic construction of Kähler-Einstein metrics with negative Ricci curvature in [10, as well as a variational approach towards a proof of the remaining main open problem concerning positive Ricci curvature. As demonstrated in [15] this approach settles the problem in the case of log Fano curves, but in the general case it hinges an a conjectural energy bound. Interestingly, as explained in [15], already the simplest case of a log Fano curve with trivial automorphism group - the complex projective line decorated with 3 weighted points - exhibits some rather intruiging connections 
between this probabilistic approach and the classical theory of hypergeometric functions and integrals, as well as Conformal Field Theory and Integrable Systems.

There are also many other connections to other fields that will not be covered here. Motivations from Quantum Gravity are discussed in [7, where a heuristic argument for the large $N$-convergence was first given. The connections to pluripotential theory and interpolation theory in complex affine space $\mathbb{C}^{n}$ are covered in [14, 47] and relations to stochastic gradient flows and optimal transport (via tropicalization) appear in [13. Moreover, connections to Arithmetic Geometry and Non-Archimidean Geometry will be developed elsewhere, as well as relations to the AdS/CFT correspondence in theoretical physics, connecting geometry to Conformal Field Theory [24].

Finally, a caveat: "The probabilistic approach to Kähler-Einstein metrics" will, in the following, refer to the approach, based on random point processes with $N$ points, introduced in 17, 10, 11]. It should, however, be pointed out that there is also a different probabilistic approach to Kähler geometry, introduced in [50], using random elements in the symmetric spaces $S L(N, \mathbb{C}) / S U(N)$. In the case of a a complex curve, there are also, as explained in [15], some relations between the canonical random point processes and the theory of Gaussian Multiplicative Chaos, as used in the probabilistic approach to Liouville Quantum Gravity in 63. It should also be pointed out that a statistical mechanics approach has previously been applied to conformal geometry in [60], which is related to the present complex-geometric setup in the case of complex curves.

\section{Contents}

1. Introduction

2. Recap of Kähler-Einstein metrics and motivation

3. A bird's-eye view of the probabilistic approach to Kähler-Einstein metrics

4. Background

5. The thermodynamical formalism

6. The large $N$-limit in the case of positive $\beta$

7. Towards the case of negative $\beta$

References

1.1. Organization. Since this work is mainly aimed to be expository, most of the proofs are merely sketched. However, there are also some new results (Theorems [3.7, 3.15, , 7.6, 7.9] ) where more details are provided.

After a brief recap of Kähler-Einstein metrics in Section 2 and some motivation we give, in Section 3, a bird's-eye view of the probabilistic approach to Kähler-Einstein metrics. First, the main results concerning the case when the $\operatorname{sign} \beta$ of the canonical line bundle $K_{X}$ is positive are explained. Then, the conjectural picture concerning the Fano setting, where $\beta<0$, is described. In Section 4 background material is provided on complex geometry, pluripotential theory, probability and variational analysis. This material is needed for the more detailed view of the probabilistic approach, which is the subject of the subsequent sections, starting in Section 5 with a thermodynamical formalism for Kähler-Einstein metrics. The focus in this section is on the analytical properties of the free energy functional (which appears as the rate functional of a large deviation principle for the canonical point processes). The connection 
to the standard functionals in Kähler geometry, in particular the Mabuchi functional is also explained. In the following Section 6 we explain the proof of the convergence of the point processes and the general large deviation principle in the case $\beta>0$. The Fano setting, where $\beta<0$, is considered in Section 7 where a a variational approach is proposed for proving the conjectural convergence of the point processes and relations to phase transitions are explored.

1.2. Acknowledgements. I am greatful to Shing-Tung Yau for the invitation to contribute to the upcoming volume of Surveys in Differential Geometry, on the occasion of his 70th birthday - his work has been a constant source of inspiration. It is also a pleasure to thank Sébastien Boucksom, David Witt-Nyström, Vincent Guedj and Ahmed Zeriahi for the stimulating collaborations [16, 17, 18, which paved the way for the current probabilistic approach. Also thanks to Daniel Persson and the referee for comments on the paper. This work was supported by grants from the KAW foundation, the Göran Gustafsson foundation and the Swedish Research Council.

\section{RECAP OF KÄHLER-EINSTEIN METRICS AND MOTIVATION}

Recall that a Kähler metric $\omega$ on a compact complex manifold $X$ of dimension $n$ is said to be Kähler-Einstein if it has constant Ricci curvature:

$$
\text { Ric } \omega=-\beta \omega
$$

for some constant $\beta$. The existence of such a metric implies that the canonical line bundle $K_{X}$ of $X$ (i.e. the top exterior power of the cotangent bundle of $X$ ) has a definite sign:

$$
\operatorname{sign}\left(K_{X}\right)=\operatorname{sign}(\beta)
$$

We will be using the standard terminology of positivity in complex geometry: a line bundle is said to be positive, $L>0$, if it is ample and negative, $L<0$, if its dual. Moreover, we shall adopt the standard additive notation for tensor products of line bundles, so that the dual of $L$ is expressed as $-L$ (see Section 4.1.2). Here we will focus on the case when $\beta \neq 0$ (see [10, Section 6.1] for probabilistic aspects of the case $\beta=0$ ). Then $X$ is automatically a complex projective algebraic manifold. After a rescaling of the metric we may as well assume that $\beta= \pm 1$. In the case when $K_{X}>0$ and $K_{X}=0$ the existence of a Kähler-Einstein metric was established in the seminal works [81, 2] and [81, respectively. However, in the case when $K_{X}<0$, i.e. when $X$ is a Fano manifold, there are obstructions to the existence of a KählerEinstein metric. According to the Yau-Tian-Donaldson conjecture a Fano manifold $X$ admits a Kähler-Einstein metric iff $X$ is K-polystable (the "only if" statement holds for singular Fano varieties [9]). This an algebro-geometric notion of stability, modeled on the notion of stability in Geometric Invariant Theory. Briefly, a Fano manifold $X$ is $\mathrm{K}$-polystable iff the DonaldsonFutaki invariant $D F(\mathcal{X})$ of any normal $\mathbb{C}^{*}$-equivariant deformation $\mathcal{X} \rightarrow \mathbb{C}$ of $\left(X,-K_{X}\right)$ (called a test configuration) is non-negative and vanishes only for the test configurations $\mathcal{X}$ whose central fiber is biholomorphic to $X$. The invariant $D F(\mathcal{X})$ may be defined as a the normalized large $N$-limit of the Chow weight of the orbit of $X$ in $\mathbb{P}^{N-1}$ under a one-parameter subgroup of $S L(N, \mathbb{C})$ associated to $\mathcal{X}$. See the survey [45] for the detailed definition and further background.

When a Fano manifold $X$ admits a Kähler-Einstein metric it is uniquely determined modulo the action of the group Aut $(X)_{0}$ of all biholomorphic automorphisms of $X$ in the connected component of the identity [4]. The dichotomy non-trivial vs. trivial group Aut $(X)_{0}$ is reflected 
in the difference between K-polystability and the stronger notion of K-stability, which implies that $\operatorname{Aut}(X)_{0}=\{I\}$.

The relation between Kähler-Einstein metrics and stability, in the sense of GIT, was propounded by Yau in [82, 83] and further developed by Tian [76] and then Donaldson [42, who considered the more general setting of constant scalar curvature metrics on polarized complex compact manifolds. In the Fano case the Yau-Tian-Donaldson conjecture was settled in [32], using a logarithmic version of Aubin's continuity (and the Cheeger-Colding-Tian theory of Gromov-Hausdorff limits of Kähler manifolds). There is also a stronger form of K-stability, called uniform K-stability, introduced in [74, 75, 40, 29]. In [20] it was shown by a direct variational approach that a Fano manifold admits a unique Kähler-Einstein -metric iff $X$ is uniformly K-stable, using pluripotential theory (including some Non-Archimidean aspects). Very recently the variational approach in [20] has also been extended to general singular Fano varieties in [66] and [65], using a notion of equivariant uniform K-stability.

2.1. The lack of explicit formulas. Already in the case when the canonical line bundle $K_{X}$ is positive and thus $X$ admits a Kähler-Einstein metric $\omega_{K E}$ with negative Ricci curvature, there are very few cases where $\omega_{K E}$ can be written down explicitly. For example, let $X$ be the projective algebraic manifold defined by the zero-locus of a homogeneous polynomial of degree $d$ in $(n+1)$-dimensional complex projective space $\mathbb{P}_{\mathbb{C}}^{n+1}$. Then $K_{X}>0$ iff $d>(n+2)$ (by the adjunction formula). However, even in the case of a complex algebraic curve $X$ in $\mathbb{P}_{\mathbb{C}}^{2}$ the problem of explicitly describing the Kähler-Einstein metric on $X$ is, in general, intractable. By the classical uniformization theory this problem is equivalent to finding an explicit biholomorphic map from $X$ to the quotient $\mathbb{H} / G$ of the upper half-plane by a discrete subgroup $G \subset S L(2, \mathbb{R})$. This has only been achieved for very special curves, using techniques originating in the classical works by Weierstrass, Riemann, Fuchs, Schwartz, Klein, Poincaré,... [72]. See, for example, [5] for the case when $X$ is a Fermat curve of degree $d \geq 4$, 48, for the case when $X$ is the Klein quartic, including arithmetic aspects and [46] for connections to the mirror-moonshine conjecture of Lian-Yau. A recurrent theme in all these cases is that the uniformizing map from $X$ to $\mathbb{H} / G$ may be expressed as a quotient of hypergeometric functions.

Thus one motivation for the probabilistic approach to Kähler-Einstein metrics is that for any given projective manifold $X$ with $K_{X}>0$ it leads to canonical approximations $\omega_{k}$ of the Kähler-Einstein metric $\omega_{K E}$ on $X$, which are explicitly expressed in terms of algebro-geometric data on $X$. This will be explained in detail in the following sections (see Corollary [3.2). For the moment we just point out that, realizing $X$ as an algebraic subvariety of $\mathbb{P}_{\mathbb{C}}^{m}$, the Kähler potential $\varphi_{k}$ on $X$ may be explicitly expressed as follows, in terms of homogeneous coordinates $z \in \mathbb{C}^{m+1}$ on $\mathbb{P}_{\mathbb{C}}^{m}$ :

$$
e^{\varphi_{k}(z)}=\frac{1}{Z_{N_{k}}} \int_{X^{N_{k}-1}} \frac{\left|P_{N_{k}}\left(z, z_{2} \ldots, z_{N_{k}}\right)\right|^{2 / k}}{|z|^{2}\left|z_{2}\right|^{2} \cdots\left|z_{N_{k}}\right|^{2}} d V^{\otimes\left(N_{k}-1\right)}
$$

where $N_{k}$ is a sequence tending to infinity (the plurigenera of $X$ ), $P_{N_{k}}$ is a homogeneous polynomial on the $N_{k}$-fold product $X^{N_{k}}$ of $X$, naturally attached to the degree $k$ component of graded homogeneous coordinate ring of $X$ and $d V$ is an algebraic volume form on $X$, induced by the embedding in $\mathbb{P}^{m}$. The normalizing constant $Z_{N_{k}}$ is given by $Z_{N, \beta}$ for $\beta=1$ where 
$Z_{N, \beta}$ is the following Archimedean zeta function 1

$$
Z_{N, \beta}:=\int_{X^{N_{k}}}\left(\frac{\left|P_{N_{k}}\left(z_{1}, z_{2} \ldots, z_{N_{k}}\right)\right|^{2 / k}}{\left|z_{1}\right|^{2}\left|z_{2}\right|^{2} \cdots\left|z_{N_{k}}\right|^{2}}\right)^{\beta} d V^{\otimes N_{k}} .
$$

In the case when $X$ is Fano the exponent $2 / k$ in formula 2.2 is replaced by $2 \beta / k$ for $\beta=-1$. The conjectural convergence towards the potential of a Kähler-Einstein metric, when $N_{k} \rightarrow \infty$, then turns out to be related to analytic properties of the corresponding Archimedean zeta function $\beta \mapsto Z_{N, \beta}$.

Incidentally, the integral in formula 2.3 is reminiscent of the Euler integral (period) formulas for the hypergeometric functions that appear in the classical uniformization theory for complex curves and integrable systems, alluded to a above. Indeed, the ordinary (Gauss) hypergeometric function with real parameters $(a, b, c)$ may be expressed as follows when $z$ is in the upper half-plane of $\mathbb{C}$ :

$$
F(z)=\frac{1}{B(b, c-b)} \int_{[0,1]}(1-z x)^{-a} x^{b-1}(1-x)^{c-b-1} d x
$$

where $B(p, q)$ is the Beta function:

$$
B(p, q)=\int_{[0,1]} x^{p-1}(1-x)^{q-1} d x, \quad \operatorname{Re} p>0, \operatorname{Re} q>0
$$

These connections can be made more precise in the case of complex curves, as explained in 15.

Remark 2.1. When $X$ is defined over $\mathbb{Q}$ the integrals in 2.3 and 2.4 are examples of periods, as defined in [62, Chapter 4], i.e. integrals of the form $\int_{\gamma} \eta$ for an algebraic form $\eta$ of maximal degree on a projective variety $Y$ defined over $\mathbb{Q}$ and homology class $\gamma \in H(Y(\mathbb{C}), \mathbb{Q})$ with boundary on a normal crossing divisor in $Y(\mathbb{C})$. Indeed, $Y(\mathbb{C})$ can be taken as the complexification of $X$, when $X$ is viewed as a real manifold of real dimension $2 n$ and $\gamma$ as $X$. As stressed in [62, in many classical cases period integrals satisfy differential equations with respect to variations of the parameters of the integrand (notably Picard-Fuch equations, such as the hypergeometric equation satisfied by $F(z)$ above). In the present setup the role of the differential equation is thus - loosely speaking - played by the Kähler-Einstein equation, but it only arises in the limit when $N \rightarrow \infty$.

The canonical Kähler potentials $\varphi_{k}$ in formula 2.2 are also somewhat reminiscent of the sequence of balanced metrics introduced in 44] (when either $K_{X}>0$ or $K_{X}<0$ ), defined as fixed point of an algebraic iteration. But one virtue of the present setup is that $\varphi_{k}$ is given by an explicitly formula.

\section{A BIRD'S-EYE VIEW OF THE PROBABILISTIC APPROACH TO KÄHLER-EINSTEIN METRICS}

We continue with the notation introduced in Section 2, with $X$ denoting a compact complex manifold with the property that its canonical line bundle $K_{X}$ has a definite sign $\beta$. First recall that it follows directly from the basic formula for the Ricci curvature of a Kähler metric,

\footnotetext{
${ }^{1}$ Integrals of complex powers of algebraic functions are Archimedean analogs of local Igusa zeta functions, defined in a p-adic setting [56].
} 
that, in the case when $\beta \neq 0$, a Kähler-Einstein metric $\omega_{K E}$ on $X$ can be recovered from its (normalized) volume form $d V_{K E}$

$$
\omega_{K E}=\frac{1}{\beta} d d^{c} \log d V_{K E},
$$

using the notation $d d^{c}:=\frac{i}{2 \pi} \partial \bar{\partial}$ (see Section 4.1). Accordingly, the strategy of the probabilistic approach is to first construct the canonical normalized volume form $d V_{K E}$ by a canonical sampling procedure on $X$.

We will denote by $\mathcal{P}(X)$ the space of all normalized measures $\mu$ on $X$, i.e. the space of all probability measures on $X$ (see Section 4.3 for a recap of the basic probabilistic background).

3.1. The case $\beta>0$. Let $X$ be a compact complex manifold with positive canonical line bundle $K_{X}$. The starting point of the probabilistic approach to Kähler-Einstein metrics is the observation that, when $K_{X}>0$, there is canonical way of sampling configurations of $N$ random points on $X$, i.e. there is a canonical random point process on $X$ with $N$ points. This means that there is a canonical sequence of symmetric probability measures $\mu^{(N)}$ on the $N$-fold products $X^{N}$ and, as shown in [10, the corresponding empirical measure

$$
\delta_{N}:=\frac{1}{N} \sum_{i=1}^{N} \delta_{x_{i}}: X^{N} \rightarrow \mathcal{P}(X)
$$

viewed as a random measure on the probability space $\left(X^{N}, \mu^{(N)}\right)$, converges in probability as $N \rightarrow \infty$, to the volume form $d V_{K E}$ of the unique Kähler-Einstein metric $\omega_{K E}$. In fact, the canonical sequence of probability measures on $\mu^{(N)}$ on $X^{N_{k}}$ is defined for a specific subsequence of integers $N$ tending to infinity, the plurigenera of $X$ :

$$
N_{k}:=\operatorname{dim} H^{0}\left(X, k K_{X}\right),
$$

where $H^{0}\left(X, k K_{X}\right)$ denotes the complex vector space of all global holomorphic sections $s^{(k)}$ of the $k$ th tensor power of the canonical line bundle $K_{X} \rightarrow X$ (aka pluricanonical forms). We recall that, in terms of local holomorphic coordinates $z \in \mathbb{C}^{n}$ on $X$, this simply means that a section $s^{(k)}$ may be represented by local holomorphic functions $s^{(k)}$ on $X$, such that $\left|s^{(k)}\right|^{2 / k}$ transforms as a density on $X$, i.e. defines a measure on $X$. The canonical probability measure $\mu^{\left(N_{k}\right)}$ on $X^{N_{k}}$ is now defined by

$$
\mu^{\left(N_{k}\right)}:=\frac{1}{Z_{N_{k}}}\left|\operatorname{det} S^{(k)}\right|^{2 / k}, Z_{N}:=\int_{X^{N_{k}}}\left|\operatorname{det} S^{(k)}\right|^{2 / k}
$$

where $\operatorname{det} S^{(k)}$ is the holomorphic section of the canonical line bundle $\left(k K_{X^{N_{k}}}\right)$ over $X^{N_{k}}$, defined by the Slater determinant

$$
\left(\operatorname{det} S^{(k)}\right)\left(x_{1}, x_{2}, \ldots, x_{N}\right):=\operatorname{det}\left(s_{i}^{(k)}\left(x_{j}\right)\right)
$$

in terms of a given basis $s_{i}^{(k)}$ in $H^{0}\left(X, k K_{X}\right)$. Note that under a change of bases the section $\operatorname{det} S^{(k)}$ only just changes by a multiplicative complex constant $c$ (the determinant of the change of bases matrix). Hence, by homogeneity, the probability measure $\mu^{\left(N_{k}\right)}$ is independent of the choice of bases and thus defines a canonical symmetric probability measure on $X^{N_{k}}$, as desired. Moreover, the probability measure is $\mu^{\left(N_{k}\right)}$ is encoded by algebro-geometric data in the following sense: using the Kodaira embedding theorem to realize $X$ as projective algebraic 
subvariety the density $\operatorname{det} S^{(k)}$ can be identified with a homogeneous polynomial (see Section 4.1.3).

The following convergence result was shown in [10:

Theorem 3.1. Let $X$ be a compact complex manifold with positive canonical line bundle $K_{X}$. Then the empirical measures $\delta_{N_{k}}$ of the corresponding canonical random point processes on $X$ converge in probability, as $N_{k} \rightarrow \infty$, towards the normalized volume form $d V_{K E}$ of the unique Kähler-Einstein metric $\omega_{K E}$ on $X$.

In particular, the convergence in probability in the previous theorem implies that the measure on $X$ defined by the expectations $\mathbb{E}\left(\delta_{N_{k}}\right)$ of the empirical measure $\delta_{N_{k}}$ converge towards $d V_{K E}$ in the weak topology of measures on $X$ :

$$
\mathbb{E}\left(\delta_{N_{k}}\right)=\int_{X^{N_{k}-1}} \mu^{\left(N_{k}\right)} \rightarrow d V_{K E}, \quad k \rightarrow \infty
$$

Noting that

$$
\omega_{k}:=d d^{c} \log \mathbb{E}\left(\delta_{N_{k}}\right)=d d^{c} \log \int_{X^{N_{k}-1}}\left|\operatorname{det} S^{(k)}\right|^{2 / k}
$$

defines a canonical sequence of Kähler metrics on $X$ (for $k$ sufficiently large) we thus arrive at the following

Corollary 3.2. Let $X$ be a compact complex manifold with positive canonical line bundle $K_{X}$. Then the canonical sequence of Kähler metrics $\omega_{k}$ converges towards the unique KählerEinstein metric $\omega_{K E}$ on $X$, in the weak topology.

It does not seem clear how to directly prove the convergence of the measures $\mathbb{E}\left(\delta_{N_{k}}\right)$ on $X$ (and the Kähler metrics $\omega_{k}$ ) without first proving the stronger convergence in probability in Theorem 3.1. Moreover, as explained in Section 6, the convergence in probability is shown to hold in the stronger exponential sense of a Large Deviation Principle (LDP). Endowing the space $\mathcal{P}(X)$ of all probability measures on $X$ by a metric compatible with the weak topology and denoting by $B_{\epsilon}(\mu)$ the ball of radius $\epsilon$ centered at a given element $\mu \in \mathcal{P}(X)$ this essentially means that there exists a functional $I(\mu)$ on $\mathcal{P}(X)$, called the rate functional, such that

$$
\operatorname{Prob}\left(\frac{1}{N} \sum_{i=1}^{N} \delta_{x_{i}} \in B_{\epsilon}(\mu)\right) \sim e^{-N I(\mu)}
$$

when first $N \rightarrow \infty$ and then $\epsilon \rightarrow 0$ (see Section 4.3 .1 for the precise meaning of a LDP). Moreover, the rate functional $I(\mu)$ is non-negative and vanishes iff $\mu=d V_{K E}$ In fact, if $\omega$ is a Kähler form in the first Chern class $c_{1}\left(K_{X}\right)$, then the rate functional $I$ may be identified with the the Mabuchi functional on the space of Kähler metrics:

$$
I\left(\frac{\omega^{n}}{\int_{X} \omega^{n}}\right)=\mathcal{M}(\omega)
$$

where $\mathcal{M}(\omega)$ denotes Mabuchi functional on the space of Kähler metrics in $c_{1}\left(K_{X}\right)$. Once the LDP 3.3 has been established the convergence in probability in Theorem 3.1 then follows from the well-known fact in Kähler geometry that $\omega_{K E}$ is unique minimizer of $\mathcal{M}$. More precisely, since the rate functional $I(\mu)$ is defined on the whole space of probability measure $\mathcal{P}(X)$ and not only on the dense subspace of volume forms, some additional arguments are required, using variational calculus on $\mathcal{P}(X)$ (see Theorem 5.4). 
3.1.1. The proof of the $L D P$ for $\beta>0$ and statistical mechanics. Fixing a volume form $d V$ on $X$ the canonical probability measure 3.1 may be expressed as

$$
\mu^{(N)}=\frac{1}{Z_{N_{k}}}\left\|\operatorname{det} S^{(k)}\right\|^{2 / k} d V^{\otimes N}
$$

where $\|\cdot\|$ denotes the metric on $K_{X}$ induced by the volume form $d V$. The starting the point of the proof of the LDP 3.3 is to rewrite this expression as a Gibbs measure:

$$
\mu_{\beta}^{\left(N_{k}\right)}=\frac{e^{-\beta N E^{(N)}}}{Z_{N, \beta}} d V^{\otimes N}, \quad Z_{N, \beta}:=\int_{: X^{N}} e^{-\beta N E^{(N)}} d V^{\otimes N}
$$

with

$$
E^{(N)}:=-\frac{1}{k N} \log \left\|\operatorname{det} S^{(k)}\left(x_{1}, \ldots, x_{N_{k}}\right)\right\|^{2}, \beta=1
$$

In the general terminology of statistical mechanics, if $X$ is a Riemannian manifold (where we assume that the Riemannian volume form $d V$ is normalized) and $E^{(N)}$ is a given symmetric function on $X^{N}$, called the energy per particle, the Gibbs measure 3.4 represents the microscopic equilibrium state of $N$ interacting identical particles on $X$ at inverse temperature $\beta$. The normalizing constant $Z_{N, \beta}$ is called the partition function.

The proof of the LDP is inspired by the notion of a mean field approximations in physics. Briefly, the idea is to first show that there exists a functional $E$ on $\mathcal{P}(X)$ such that the energy per particle, may be approximated as

$$
E^{(N)}\left(x_{1}, \ldots x_{N}\right) \approx E(\mu), \quad \frac{1}{N} \sum_{i=1}^{N} \delta_{x_{i}} \approx \mu
$$

in an appropriate sense, as $N \rightarrow \infty$. Formally, this suggests that the rate functional is given by

$$
\left.\left.I(\mu)=F_{\beta}(\mu)-\inf _{\mathcal{P}(X)} F_{\beta}, \quad F_{\beta}(\mu)=\beta E(\mu)+D_{d V}(\mu) \in\right] 0, \infty\right],
$$

where $D_{d V}(\mu)$ is the entropy of $\mu$ relative to $d V$, which arises when integrating the volume $d V^{\otimes N}$ form over small balls in the $N$-particle configuration space $X^{N} / S_{N}$ (see Section 6.1).

In the general statistical mechanical setup $E(\mu)$ represents the energy of the macroscopic state $\mu$ and $F_{\beta}(\mu)$ its free energy, at inverse temperature $\beta$. In the present setting the role of the macroscopic energy $E(\mu)$ is played by the pluricomplex energy of the measure $\mu$ (introduced in [18]), defined with respect to the Kähler form -Ric $d V$. More generally, the same proof yields the following general result, where the role of $K_{X}$ is played by a given positive line bundle $L$ over a compact complex manifold $X$.

Theorem 3.3. Let $L$ be a positive line bundle over a compact complex manifold $(X, L)$. Given a volume form $d V$ on $X$ and a metric $\|\cdot\|$ on $L$ denote by $\mu_{\beta}^{(N)}$ the corresponding Gibbs measure 3.4. at inverse temperature $\beta \in] 0, \infty[$. Then the laws of the corresponding random measures $\delta_{N}$ on $\left(X^{N}, \mu_{\beta}^{(N)}\right)$ satisfy a Large Deviation Principle (LDP) with speed $N$ and rate functional $F_{\beta}(\mu)-C_{\beta}$, where

$$
F_{\beta}(\mu)=\beta E_{\omega_{0}}(\mu)+D_{d V}(\mu), C_{\beta}=\inf _{\mathcal{P}(X)} F_{\beta}
$$


As a consequence, the empirical measure $\delta_{N}$ converges in probability towards the unique minimizer $\mu_{\beta}$ of the free energy functional $F_{\beta}$ on $\mathcal{P}(X)$, which is the volume form characterized by the property that the Kähler metric

$$
\omega_{\beta}:=\omega_{0}+\frac{1}{\beta} d d^{c} \log \frac{\mu_{\beta}}{d V}
$$

is the unique solution to the twisted Kähler-Einstein equation

$$
\operatorname{Ric} \omega=-\beta \omega+\theta, \quad \theta:=\beta \omega_{0}+\operatorname{Ric} d V .
$$

In particular, specializing the previous theorem to the "canonical case" $L=K_{X}, \omega_{0}:=-\operatorname{Ric} d V$ and $\beta=1$ yields Theorem 3.1. The technical ingredients in the proof of the LDP in Theorem 3.3 are discussed in Section 6. An important feature of the case $\beta>0$ is that $\beta E^{(N)}$ is quasisuperharmonic. However, allowing negative values of $\beta$ is crucial in the case when $X$ is a Fano manifold, as discussed below.

3.1.2. Varieties of positive Kodaira dimension and log pairs. Before turning to Fano manifolds we recall that, as shown in [11], Theorem 3.1 holds in much greater generality. Indeed, the minimal requirement for the previous setup to apply is that the plurigenera $N_{k}$ of $X$ tend to infinity as, $k \rightarrow \infty$. In the classical terminology of algebraic geometry this means that $K_{X}$ has strictly positive Kodaira dimension $\kappa$ and is shown in [11] the analog of Theorem 3.1 then holds if $d V_{K E}$ is replaced by the canonical measure on $X$ first introduced in [78, 73] in different contexts. Then $\omega_{k}$ (defined by formula 3.2) defines a canonical sequence of positive currents in $c_{1}\left(K_{X}\right)$ which are Kähler on the complement in $X$ of the base locus defined by $k K_{X}$ and $\omega_{k}$ converges in the weak sense of currents to the pull-back to $X$ of the canonical twisted Kähler-Einstein current on the $\kappa$-dimensional base $Y$ of the litaka fibration $X \rightarrow Y$, whose generic fibers are Calabi-Yau manifolds of dimension $n-\kappa$.

In fact, these results hold more generally when $X$ is a (normal) projective algebraic variety (with klt singularities) and may be formulated in a birationally invariant manner using the general the setting of log pairs $(X, \Delta)$, in the usual sense of MMP [61. Recall that a log pair $(X, \Delta)$ is a complex (normal) variety $X$ endowed with a $\mathbb{Q}$-divisor $\Delta$ on $X$, i.e. a sum of irreducible subvarieties of $X$ of codimension one, with coeffients $w_{i}$ in $\mathbb{Q}$. Then the role of the canonical line bundle $K_{X}$ is placed by the log canonical line bundle

$$
K_{(X, \Delta)}:=K_{X}+\Delta
$$

(when defined as a $\mathbb{Q}$-line bundle) and the role of the Ricci curvature Ric $\omega$ of a metric $\omega$ is played by twisted Ricci curvature Ric $\omega-[\Delta]$, where $[\Delta]$ denotes the current of integration defined by $\Delta$. The corresponding log Kähler-Einstein equation reads

$$
\operatorname{Ric} \omega-[\Delta]=\beta \omega,
$$

where $[\Delta]$ denotes the current of integration along $\Delta$. When $\beta$ is non-zero existence of a solution $\omega_{K E}$ forces

$$
\beta\left(K_{X}+\Delta\right)>0
$$

In general, the equation 3.6 should be interpreted in the weak sense of pluripotential theory [49, 19]. However, in the $\log$ smooth case it follows from [57, 55] that a positive current $\omega$ solves the equation [3.6] iff $\omega$ is a bona fide Kähler-Einstein metric on $X-\Delta$ and $\omega$ has edge-cone singularities along $\Delta$, with cone-angle $2 \pi\left(1-w_{i}\right)$, prescribed by the coefficents $w_{i}$ of $\Delta$. 
Remark 3.4. Starting with a normal variety $Y$ such that $K_{Y}$ is defined as a $\mathbb{Q}$-line bundle and taking $X$ to be a non-singular resolution of $Y, X \rightarrow Y$, the pull-back of $K_{Y}$ to $X$ is of the form $K_{(X, \Delta)}$ for an exceptional divisor $\Delta$ on $X$ that may be assumed to have simple normal crossings. The divisor $\Delta$ is said to be klt (Kawamata Log Terminal) if all of its coefficients $w_{i}$ satisfy $w_{i}<1$ 61. If this is the case the variety $Y$ is also said to have klt singularities. In another direction, if $Y$ is a projective algebraic manifold with positive (but not maximal) Kodaira dimension, then $K_{Y}$ is the pull-back of $K_{(X, \Delta)}$ for a klt divisor $\Delta$ supported on the ramification locus of the litaka fibration $Y \rightarrow X$.

As shown in [11] Theorem 3.1 can be generalized to any klt pair $(X, \Delta)$ such that $(X, \Delta)$ has positive Kodaira dimension.

3.2. The case $\beta<0$. When $K_{X}$ is negative, that is, $X$ is a Fano manifold, we replace the zero-dimensional spaces $H^{0}\left(X, k K_{X}\right)$ with the spaces $H^{0}\left(X,-k K_{X}\right)$ of dimension

$$
N_{k}:=\operatorname{dim} H^{0}\left(X,-k K_{X}\right),
$$

tending to infinity, as $k \rightarrow \infty$. We are then forced to replace the power $2 / k$ in formula 3.1 with a negative power $-2 / k$ in order to ensure that

$$
\left|\operatorname{det} S^{(k)}\right|^{-2 / k}
$$

transforms as a density on $X^{N_{k}}$, i.e. defines a global measure on $X^{N_{k}}$. However, then the corresponding normalization constant $Z_{N_{k}}$

$$
Z_{N_{k}}:=\int_{X^{N_{k}}}\left|\operatorname{det} S^{(k)}\right|^{-2 / k}
$$

may diverge, since the integrand blows-up along the zero-locus in $X^{N_{k}}$ of the section $\operatorname{det} S^{(k)}$. Accordingly, we will say that a Fano manifold $X$ is Gibbs stable at level $k$ if $Z_{N_{k}}<\infty$ and Gibbs stable if it is Gibbs stable at level $k$ for $k$ sufficiently large. For a Gibbs stable Fano manifold $X$ we set

$$
\mu^{\left(N_{k}\right)}:=\frac{1}{Z_{N_{k}}}\left|\operatorname{det} S^{(k)}\right|^{-2 / k},
$$

which defines a canonical symmetric probability measure on $X^{N_{k}}$, i.e. a canonical random point process on $X$ with $N_{k}$ points. We thus arrive at the following probabilistic analog of the Yau-Tian-Donaldson conjecture

Conjecture 3.5. Let $X$ be Fano manifold. Then

- $X$ admits a unique Kähler-Einstein metric $\omega_{K E}$ if and only if $X$ is Gibbs stable.

- If $X$ is Gibbs stable, the empirical measures $\delta_{N}$ of the corresponding canonical point processes converge in probability towards the normalized volume form of $\omega_{K E}$.

If $X$ is Gibbs stable then

$$
\omega_{k}:=-d d^{c} \log \mathbb{E}\left(\delta_{N_{k}}\right)=-d d^{c} \log \int_{X^{N_{k}-1}}\left|\operatorname{det} S^{(k)}\right|^{-2 / k}
$$

defines a sequence of canonical positive currents (as follows from the positivity of direct image bundles in [25]; see [11, Prop 6.5]). In analogy with Corollary 3.2 it seems natural to also conjecture that, if $X$ is Gibbs stable, then $\omega_{k}$ converges to a Kähler-Einstein metric on $X$. 
It should be stressed that the Gibbs stability of $X$ implies that the group Aut $(X)_{0}$ is trivial [11, Prop 6.5]. Accordingly, when comparing Conjecture 3.5 with the Yau-Tian-Donaldson conjecture one should view Gibbs stability as the analog of K-stability.

In the light of Theorem 6.2 it is natural to pose the following stronger LDP form of the previous conjecture:

Conjecture 3.6. Let $X$ be a Fano manifold. Then $X$ admits a unique Kähler-Einstein metric iff the canonical measure $\mu^{(N)}$ is a probability measure for $N$ sufficiently large. Moreover, if this is the case then the laws of the empirical measures $\delta_{N}$ on $\left(X^{N}, \mu^{(N)}\right)$ satisfy a Large Deviation Principle $(L D P)$ with speed $N$ and rate functional $F_{-1}(\mu)-C_{\beta}$, where

$$
F_{-1}(\mu)=-E_{\omega_{0}}(\mu)+D_{d V}(\mu), C_{-1}=\inf _{\mathcal{P}(X)} F_{-1} .
$$

To highlight the connection to the LDP in Theorem 6.2, fix a volume form $d V$ on $X$ and denote by $\|\cdot\|$ the induced metric on the anti-canonical line bundle $-K_{X}$. Then the canonical probability measure 3.8 may be expressed as

$$
\mu_{\beta}^{(N)}:=\frac{1}{Z_{N_{k}, \beta}}\left\|\operatorname{det} S^{(k)}\right\|^{2 \beta / k} d V^{\otimes N}, \quad Z_{N_{k}, \beta}:=\int_{X^{N_{k}}}\left\|\operatorname{det} S^{(k)}\right\|^{2 \beta / k} d V^{\otimes N},
$$

for $\beta=-1$. Since the rate functional of a LDP is automatically lower semi-continuous (lsc) the validity of the LDP in Conjecture 3.6 would imply that the free energy functional $F_{-1}$ is lsc on $\mathcal{P}(X)$. This is indeed the case. More precisely, the following result holds, deduced from a combination of results in [76, 19]:

Theorem 3.7. Let $X$ be a Fano manifold and set $\beta=-1$. Then the free energy functional $F_{\beta}$ on the space $\mathcal{P}(X)$ of probability measures on $X$ is lower semi-continuous iff $X$ admits a unique Kähler-Einstein metric $\omega_{K E}$. Moreover, if $F_{\beta}$ is lsc, then the normalized volume form of $\omega_{K E}$ is the unique minimizer of $F_{\beta}$.

We note in passing that, just as in the case when $K_{X}>0$ and $\beta=1$, the free energy functional $F_{\beta}$ at $\beta=-1$, restricted to the space of volume forms on $\mathcal{P}(X)$, may be identified with the Mabuchi functional $\mathcal{M}$ on $c_{1}\left(-K_{X}\right)$.

3.2.1. Symmetry breaking. As discussed above, Gibbs stability should be considered as the analog of K-stability. One is thus naturally lead to ask whether there is also a notion of "Gibbs polystability", taking the action of the group Aut $(X)_{0}$ into account? This is an intriguing question that we shall sidestep here, by breaking the Aut $(X)_{0}$-symmetry as follows. Fixing a volume form $d V$ one can viewed the probability measure $\mu_{\beta}^{(N)}$, defined by formula 3.10, as a deformation of the canonical measure $\mu^{(N)}$ to $\beta<-1$. Since $-K_{X}>0$ we may pick a volume form $d V$ inducing a metric on $-K_{X}$ with positive curvature:

$$
\omega_{0}:=\operatorname{Ric} d V>0
$$

One advantage of allowing $\beta>-1$ is that it attenuates the singularities of the integrand, as further discussed below.

Conjecture 3.8. Assume that $X$ is a Fano manifold. If $X$ admits a Kähler-Einstein metric, then for any given $\beta<1$ we have that $Z_{N_{k}, \beta}<\infty$ for $k$ sufficiently large. Moreover, the empirical measure $\delta_{N}$ on $\left(X^{N_{k}}, \mu_{\beta}^{\left(N_{k}\right)}\right)$, convergence in law towards a volume form $\mu_{\beta}$ and

$$
\lim _{\beta \rightarrow} \mu_{\beta}=d V_{K E}
$$


where $d V_{K E}$ is the volume form of a Kähler-Einstein metric on $X$. More precisely, for $\beta>-1$

$$
\omega_{\beta}:=\operatorname{Ricd} V+\beta^{-1} d d^{c} \log \frac{\mu_{\beta}}{d V}
$$

is the unique Kähler metric solving

$$
\operatorname{Ric} \omega_{\beta}=-\beta \omega_{\beta}+(1+\beta) \operatorname{Ric} d V,
$$

One motivation for this conjecture is that it holds for $\beta>0$. Indeed, according to Theorem 3.3 the result holds in the stronger sense of large deviations. As a consequence, for $\beta>0$, the LDP also implies that

$$
-\lim _{N \rightarrow \infty} \frac{1}{N} \log Z_{N, \beta}=\inf _{\mathcal{P}(X)} F_{\beta},
$$

if the fixed bases in $H^{0}\left(X,-k K_{X}\right)$ is taken to be orthonormal with respect to the scalar product induced by $d V$.

Remark 3.9. Incidentally, the equation 3.11 coincides with the one introduced by Aubin's in his continuity method for solving the Kähler-Einstein equation at $\beta=-1$. [3] In Aubin's notation the time-parameter corresponds to $-\beta \in[0,1]$. The uniqueness of solutions for $\beta>-1$ was established in [4]. Moreover, it was also shown in [4] that if $X$ admits a Kähler-Einstein metric, then $\omega_{\beta}$ exists for any $\beta>-1$ and converges, as $\beta \rightarrow-1$, towards a particular Kähler-Einstein metric, singled out by $d V$.

From a statistical mechanics point of view it may, at a first glance, seem rather odd to consider the case when $\beta<0$, since it would correspond to a negative (absolute!) temperature. But the notion of negative temperature states does make sense physically (see the discussion in [14, Remark 8.1]). Moreover, from an equivalent point of view we may as well consider the case of unit temperature and instead replace the energy particle $E^{(N)}$ with the rescaled energy $\beta E^{(N)}$ (thus treating $\beta$ as a coupling constant). For $\beta>0$ this energy is is repulsive, since it tends to $\infty$ as any two particle positions merge (due to the vanishing of the determinant $\operatorname{det} S^{(k)}\left(x_{1}, \ldots, x_{N_{k}}\right)$ ). However, when $\beta$ changes sign, from positive to negative, the rescaled energy $\beta E^{(N)}$ becomes attractive; it tends to $-\infty$ as any two points merge. Still, it turns out that the system is sufficiently mildly attractive to allow $Z_{N, \beta}$ to be finite for $\beta>\beta_{0}$, for some negative $\beta_{0}$, ensuring that the Gibbs measure $\mu_{\beta}^{(N)}$ is well-defined. This may be interpreted as a statistical mechanical type of stability, since it amounts to the existence of the microscopic $N$-particle equilibrium state. More precisely, there exists $\left.\beta_{0} \in\right]-\infty, 0[$ such that for any $\beta>\beta_{0}$

as $N \rightarrow \infty$.

$$
\frac{1}{N} \log Z_{N, \beta} \geq-C_{\beta}
$$

3.2.2. Stability thresholds and uniform Gibbs stability. The previous discussion motivates the introduction of the following "microscopic stability thresholds"

$$
\gamma_{N_{k}}(X):=\sup \left\{\gamma: Z_{N_{k},-\gamma}<\infty\right\}
$$


and their limit

$$
\gamma(X):=\liminf _{k \rightarrow \infty} \gamma_{N_{k}}
$$

as well as the "macroscopic stability threshold"

$$
\Gamma(X):=\sup _{\beta>0}\left\{-\beta: \inf _{\mathcal{P}(X)} F_{\beta}>-\infty\right\}
$$

In the "thermodynamical limit", $N \rightarrow \infty$, it is thus natural to make the following

Conjecture 3.10. Let $X$ be a Fano manifold. Then the two invariants $\gamma(X)$ of $X$ and $\Gamma(X)$, defined above, coincide:

$$
\gamma(X)=\Gamma(X)
$$

Remark 3.11. The threshold $\gamma_{N}(X)$ may be interpreted as the threshold where the selfattraction of the $N$-particle system can no longer be compensated by the disorder resulting from the randomness. Similarly, the threshold $\Gamma(X)$ is the threshold where the macroscopic tendency to self-attract and form singular states can no longer be balanced by the regularizing effect of the entropy.

Let us call a Fano manifold uniformly Gibbs stable if $\gamma(X)>1$. This should be thought of us an analog of the notion of uniform K-stability. By the results in 20] a Fano manifold is uniformly K-stable iff $\Gamma(X)>1$. Hence, the validity of the previous conjecture would imply the validity of the following one:

Conjecture 3.12. Let $X$ be a Fano manifold. Then $X$ is uniformly Gibbs stable $X$ iff $X$ is uniformly K-stable.

Remark 3.13. Combining [32] and [29, 40] reveals that a Fano manifold is, in fact, uniformly Kstable iff it is K-stable. This leads one to wonder whether Gibbs stability, is, in fact, equivalent to the uniform Gibbs stability? Theorem 3.15 below shows that for log Fano curves this is indeed the case.

As observed in [11] the notion of Gibbs stability introduced above can also be given the following purely algebro-geometric formulation in the spirit of Minimal Model Program (MMP). Let $\mathcal{D}_{k}$ be the effective divisor in $X^{N_{k}}$ cut out by the section $\operatorname{det} S^{(k)}$. Geometrically, $\mathcal{D}_{k}$ may be represented as the following incidence divisor in $X^{N_{k}}$ :

$$
\mathcal{D}_{k}:=\left\{\left(x_{1}, \ldots x_{N}\right) \in X^{N_{k}}: \exists s \in H^{0}\left(X,-k K_{X}\right): s\left(x_{i}\right)=0, i=1, \ldots, N_{k}\right\}
$$

Gibbs stability at level $k$ amounts to the anti-canonical $\mathbb{Q}$-divisor $\mathcal{D}_{k} / k$ on $X^{N_{k}}$ having klt singularities (see Remark 3.4), which means that

$$
\operatorname{lct}\left(X^{N_{k}}, \mathcal{D}_{k} / k\right)>1
$$

for $k>>1$, where $\operatorname{lct}\left(X^{N_{k}}, \mathcal{D}_{k} / k\right)$ denotes the log canonical threshold (lct) of the $\mathbb{Q}$-divisor $\mathcal{D}_{k} / k$ on $X^{N_{k}}$ [61]. Indeed, it follows from the standard analytic interpretation of the lct as an integrability threshold that

$$
\gamma_{k}(X)=\operatorname{lct}\left(X^{N_{k}}, \mathcal{D}_{k} / k\right)
$$

Using properties of log canonical and techniques from the MMP one direction of Conjecture was established in [53] (see also [51] where K-stability was first shown):

Theorem 3.14. (Fujita-Odaka) [53] Uniform Gibbs stability implies uniform K-stability 
Let us briefly recall the elegant argument in [53]. First, by [53, Thm 2.5],

$$
\gamma_{k}(X) \leq \delta_{k}(X):=\inf _{D_{k}} \operatorname{lct}\left(X, D_{k}\right)
$$

where the inf is taken over all anti-canonical $\mathbb{Q}$-divisors $D_{k}$ on $X$ of $k$-basis type, i.e. $D_{k}$ is the normalized sum of the $N_{k}$ zero-divisors on $X$ defined by the members of a given basis in $H^{0}\left(X,-K_{X}\right)$. Finally, by [53, Thm 0.3], if the invariant $\delta(X)$ defined as the limsup of $\delta_{k}(X)$ satisfies $\delta(X)>1$, then $X$ is uniformly K-stable, as follows from the valuative criterion in 64, 52, (see also [20, 28, 35] for related results).

Combining Theorem 3.14 with 32 or 20 shows that uniform Gibbs stability implies the existence of a unique Kähler-Einstein metric $\omega_{K E}$. This is in line with Conjecture 3.5. However, the converse implication is still open, as well as the problem of establishing the convergence of the corresponding canonical random point processes towards $d V_{K E}$, when it exists. Here we will focus on the convergence problem, introducing a variational approach. As shown in [21] a non-Archimedean analog of this variational approach also has bearings om the converse of Theorem 3.14.

3.2.3. A variational approach towards proving the convergence in Conjecture 3.5. As discussed in Section 7 the proof of the LDP in Theorem 3.3 brakes down when $\beta<0$. To handle this case a variational approach is proposed in Section 7 . The approach, which is based on Gibbs variational principle, reveals that it is enough to establish the asymptotics 3.12 for $\beta=-1$ :

$$
-\lim _{N \rightarrow \infty} \frac{1}{N} \log Z_{N,-1}=\inf _{\mathcal{P}(X)} F_{-1}
$$

We show that the upper bound does hold, but the lower bound hinges on a conjectural upper bound on the mean energy of the $N$-particle Gibbs measures. By making contact with the theory of phase transitions in statistical mechanics, we also observe that if there exists $\beta_{0}<-1$ and a function $f(\beta)$ on $] \beta_{0}, 0[$ such that

$$
-\lim _{N \rightarrow \infty} \frac{1}{N} \log Z_{N, \beta}=f(\beta)
$$

then the convergence 3.15 holds iff $f(\beta)$ is real-analytic. Hence, if this is the case then the convergence in Conjecture 3.5 holds. Moreover, as explained in Section 7.1, the real-analyticity in question can be related to the distribution of the poles of the Archimedean zeta functions $Z_{N, \beta}$.

3.2.4. The case of log Fano curves. There is only one-dimensional Fano manifold $X$ - the complex projective line (the Riemann sphere) - and its Kähler-Einstein metrics are all biholomorphically equivalent to the standard round metric on the two-sphere. A geometrically more interesting situation appears when introducing weighted points (conical singularities) on the Riemann sphere. From the algebro-geometric point of view this fits into the general setting of log Fano manifolds. A log pair $(X, \Delta)$, consisting of a complex manifold $X$ and an effective $\mathbb{Q}$-divisor $\Delta$, is said to be a log Fano manifold if its anti-canonical line bundle is positive, $-\left(K_{X}+\Delta\right)>0$. The corresponding $\log$ Kähler-Einstein equation 3.6 for $\beta=-1$ thus reads

$$
\operatorname{Ric} \omega=-\omega+[\Delta] \text {. }
$$

To any $\log$ Fano manifold $(X, \Delta)$ we may attach a sequence of canonical probability measures $\mu_{\Delta}^{\left(N_{k}\right)}$ on $X^{N_{k}}$ by simply replacing the anti-canonical line bundle $-K_{X}$ with $-K_{(X, \Delta)}$ and 
setting

$$
\mu_{\Delta}^{\left(N_{k}\right)}:=\frac{1}{Z_{N_{k}}}\left|\operatorname{det} S^{(k)}\left(z_{1}, \ldots, z_{N}\right)\right|^{-2 / k}\left|s_{\Delta}\right|^{-2}\left(z_{1}\right) \cdots\left|s_{\Delta}\right|^{-2}\left(z_{N_{k}}\right),
$$

which is a well-defined probability measure, as long as the corresponding normalizing constant is finite,

$$
Z_{N_{k}}:=\int_{X^{N_{k}}}\left|\operatorname{det} S^{(k)}\left(x_{1}, \ldots, x_{N_{k}}\right)\right|^{-2 / k}\left|s_{\Delta}\right|^{-2}\left(x_{1}\right) \cdots\left|s_{\Delta}\right|^{-2}\left(x_{N}\right)<\infty
$$

We then say that $\log$ Fano manifold $(X, \Delta)$ is Gibbs stable. The invariants $\gamma_{k}(X, \Delta)$ and uniform Gibbs stability of $(X, \Delta)$ can also be defined as before, mutatis mutandis.

Now, let $(X, \Delta)$ be a log Fano curve $(X, \Delta)$, i.e. $X$ is the complex projective line and

$$
\Delta=\sum_{i=1}^{m} w_{i} p_{i}
$$

for positive weights $w_{i}$ satisfying $\sum_{i=1}^{m} w_{i}<2$. In [15] it is shown that the conjectures discussed above hold for any log Fano curve:

Theorem 3.15. Let $(X, \Delta)$ be a log Fano curve. Then the following is equivalent

- $(X, \Delta)$ is Gibbs stable

- $(X, \Delta)$ is uniformly Gibbs stable

- The following weight condition holds:

$$
w_{i}<\sum_{i \neq j} w_{j}, \forall i
$$

- There exists a unique a unique Kähler-Einstein metric $\omega_{K E}$ for $(X, \Delta)$

Moreover, if any of the conditions above hold, then the laws of the random measures $\delta_{N}$ on $\left(X^{N}, \mu_{\Delta}^{(N)}\right)$ satisfy a Large Deviation Principle (LDP) with speed $N$ and a rate functional $I$ with a unique minimizer $\omega_{K E} / \int_{X} \omega_{K E}$.

In this logarithmic setting the rate functional $I$ has the property that

$$
I\left(\frac{\omega}{\int_{X} \omega}\right)=\mathcal{M}_{(X, \Delta)}(\omega)
$$

where $\mathcal{M}_{(X, \Delta)}$ denote the Mabuchi functional for $(X, \Delta)$, which in the general setting of log Fano varieties was defined in [19] on the space of finite energy currents in $\omega$ in $-c_{1}\left(K_{X}+\Delta\right)$. As explained in [15], the previous theorem is a direct consequence of the LDP in [12, Thm 1.5], concerning singular pair interactions, which generalize the vortex model in two-dimensional hydrodynamics in [31, 59]. A key ingredient is an a priori estimate on the correlation measures of the processes, which builds on [31, 59] and implies the conjectural energy bound property 7.4 in this setting.

The problem of finding constant curvature metrics on Riemann surfaces with conical singularities has a long history and was first posed as a competition topic by the Göttingen Mathematical Society in 1890 [72]. The weight condition in the previous theorem first appeared in [79, where the existence of $\omega_{K E}$ was established and the uniqueness was settled in [68. By [52, Ex. 6.6] the weight condition [3.17 is equivalent to the uniform K-stability of $(X, \Delta)$, which thus is equivalent to uniform Gibbs stability in this setting. 


\section{BACKGROUND}

4.1. Complex geometry. We start by recalling some basic complex geometry - for more background see, for example, the exposition in [14] and the books [40, 54]. Let $X$ be an $n$-dimensional compact complex manifold and denote by $J$ the corresponding complex structure, viewed as an endomorphism of the real tangent bundle satisfying $J^{2}=-I$.

4.1.1. Kähler forms/metrics. On a complex manifold $(X, J)$ anti-symmetric two forms $\omega$ and symmetric two tensors $g$ on $T X \otimes T X$, which are $J$-invariant, may be identified by setting

$$
g:=\omega(\cdot, J \cdot)
$$

Such a real two form $\omega$ is said to be Kähler if it is closed, $d \omega=0$, and the corresponding symmetric tensor $g$ is positive definite (i.e. defines a Riemannian metric)2. Conversely, a Riemannian metric $g$ is said to be Kähler if it arises in this way (in Riemannian terms this means that parallel transport with respect to $g$ preserves $J$ ). By the local $\partial \bar{\partial}-$ lemma a $J$-invariant two form $\omega$ is closed, i.e. $d \omega=0$ if and only if $\omega$ may be locally expressed as $\omega=\frac{i}{2 \pi} \partial \bar{\partial} \phi$, in terms of a local smooth function $\phi$ (called a local potential for $\omega$ ). In real notation this means that

$$
\omega=d d^{c} \phi, d^{c}:=-\frac{1}{4 \pi} J^{*} d
$$

Remark 4.1. The normalization above ensures that $d d^{c} \log |z|^{2}$ is a probability measure on $\mathbb{C}$.

We will denote by $[\omega] \in H^{2}(X, \mathbb{R})$ the de Rham cohomology class represented by $\omega$. If $\omega_{0}$ is a fixed Kähler form then, according to the global $\partial \bar{\partial}-$ lemma, any other Kähler metric in $\left[\omega_{0}\right]$ may be globally expressed as

$$
\omega_{\varphi}:=\omega_{0}+d d^{c} \varphi, \quad \varphi \in C^{\infty}(X),
$$

where $\varphi$ is determined by $\omega_{0}$ up to an additive constant and called a Kähler potential for $\omega_{\varphi}$. The space of all Kähler potentials is denoted by

$$
\mathcal{H}(X, \omega):=\left\{\varphi \in C^{\infty}(X): \omega_{\varphi}>0\right\}
$$

The association $\varphi \mapsto \omega_{\varphi}$ thus allows one to identify $\mathcal{H}(X, \omega) / \mathbb{R}$ with the space of all Kähler forms in $\left[\omega_{0}\right]$.

4.1.2. Metrics on line bundles and curvature. Let $L$ be a holomorphic line bundle on $X$ and $\|\cdot\|$ a Hermitian metric on $L$. The normalized curvature two-form of $\|\cdot\|$ may be (locally) written as

$$
\omega:=-d d^{c} \phi \log \left\|e_{U}\right\|^{2}, \quad \phi:=-\log \left\|e_{U}\right\|^{2}
$$

in terms of a given local trivialization of $L$, i.e. a non-vanishing holomorphic section $e_{U}$ of $L$ over $U \subset X$. The local function $\phi$ is called the weight of the metric. The corresponding cohomology class $[\omega]$ is independent of the metric $\|\cdot\|$ on $L$ and coincides with the first Chern class $c_{1}(L)$ in $H^{2}(X, \mathbb{R}) \cap H^{2}(X, \mathbb{Z})$ (conversely, any such cohomology class is the first Chern class of line bundle $L)$. A line bundle $L$ is said to be positive if it admits a metric with positive curvature, i.e. such that the curvature form $\omega$ is Kähler. Then the pair $(X, L)$ is called

\footnotetext{
${ }^{2} \mathrm{~A} J$-invariant two form $\omega$ is usually said to be of type $(1,1)$ since $\omega=\sum_{i, j} \omega_{i j} d z_{i} \wedge d \bar{z}_{j}$ in local holomorphic coordinates.
} 
a polarized manifold. Fixing a reference metric $\|\cdot\|_{0}$ on $L$ with curvature form $\omega_{0}$ any other metric on $L$ may be expressed as

$$
\|\cdot\|=\|\cdot\|_{0} e^{-\varphi / 2}
$$

for $\varphi \in C^{\infty}(X)$. The curvature form of $\|\cdot\|$ is thus given by $\omega_{\varphi}$ which is positive iff $\varphi \in$ $\mathcal{H}\left(X, \omega_{0}\right)$. Note that the definitions are made so that

$$
\varphi=\phi-\phi_{0}
$$

in terms of the local weights $\phi$ and $\phi_{0}$ of the metrics $\|\cdot\|$ and $\|\cdot\|_{0}$. Any positive line bundle $L$ is big, i.e. there exists $V>0$ (called the volume of $L$ ) such that

$$
N_{k}:=\operatorname{dim} H^{0}(X, k L)=V k^{n}+o\left(k^{n}\right), \quad k \rightarrow \infty
$$

This follows, for example, from the Kodaira embedding theorem (recalled below) and the volume $V$ may be expressed as

$$
V:=\int_{X} \omega_{0}^{n}
$$

Given a metric $\|\cdot\|$ on $L$ we will use the same notation $\|\cdot\|$ for the induced metric on the tensor products of $L$ over $X$, obtained by imposing that $\|\cdot\|$ be multiplicative. In particular, if $\phi$ is a local weight for $\|\cdot\|$ (defined with respect to the local trivialization $e_{U}$ ) then $k \phi$ is a local weight for the $k$ th tensor product of $L$ (defined with respect to the local trivialization $e_{U}^{\otimes k}$ ). This motivates using the additive notation $k L$ for tensor products. More generally, we will use the same notation $\|\cdot\|$ for the induced metrics on the line bundles $(k L)^{\bigotimes N}$ over the $N$-fold products $X^{N}$.

4.1.3. Algebraic embeddings of polarized manifolds. Recall that the the $m$-dimensional complex projective space $\mathbb{P}_{\mathbb{C}}^{m}$ is defined by

$$
\mathbb{P}_{\mathbb{C}}^{m}\left(:=\mathbb{C}^{m+1} / \mathbb{C}^{*}\right.
$$

Denote, as usual, by $\mathcal{O}(1)$ the hyperplane line bundle over $\mathbb{P}_{\mathbb{C}}^{m}$, i.e. the dual of the tautological line bundle $\mathbb{C}^{m+1} \rightarrow \mathbb{P}_{\mathbb{C}}^{m}$. The space $H^{0}(X, k \mathcal{O}(1))$ may be identified with the space of all homogeneous polynomials on $\mathbb{C}^{m+1}$ of degree $k$. The line bundle $\mathcal{O}(1) \rightarrow \mathbb{P}_{\mathbb{C}}^{m}$ comes with a positively curved metric, namely the Fubini-Study metric induced by the Euclidean norm on $\mathbb{C}^{m+1}$ (see [14, Section 3.7] for more background). Hence, $(X, L)$ is a polarized manifold, in the sense of the previous section. More generally, if $X$ is a complex submanifold of $\mathbb{P}_{\mathbb{C}}^{m}$ (which, by Chow's theorem, equivalently means that $X$ is a algebraic) then $\left(X, \mathcal{O}_{X}(1)\right)$ is a polarized manifold, where $\mathcal{O}(1)_{X}$ denotes the restriction of $\mathcal{O}(1) \rightarrow \mathbb{P}^{m}$ to $X$. Indeed, the restriction to $\mathcal{O}_{X}(1)$ of the Fubini-Study metric on $\mathcal{O}(1)$ is positively curved. Conversely, by the Kodaira embedding theorem, if $(X, L)$ is a polarized manifold, then after perhaps replacing $L$ by a large tensor power, $X$ may be holomorphically embedded in a projective space $\mathbb{P}^{m}$ in such a way that $L$ gets identified with $\mathcal{O}(1)_{X}$ :

$$
X \rightarrow \mathbb{P}\left(H^{0}(X, L)^{*}\right), \quad x \mapsto\left[s_{0}(x): \ldots: s_{m}(x)\right] \in \mathbb{P}^{m}
$$

where $x$ is mapped to the evaluation functional at $x$ and $s_{0}, \ldots, s_{m}$ denotes a fixed basis in $\mathbb{P}\left(H^{0}(X, L)\right.$. Thus, for $k$ sufficently large, $H^{0}(X, k L)$ identifies with the restriction to $X$ of the space of all $k$-homogeneous polynomials over $\mathbb{P}^{m}$. By Chow's theorem the embedding of $X$ is an algebraic submanifold and hence a line bundle is positive iff it is ample, in the algebro-geometric sense. 
4.1.4. The canonical line bundle and Ricci curvature. When $L$ is the canonical line bundle $K_{X}$, i.e. the top exterior power of the holomorphic cotangent bundle of $X$ :

$$
K_{X}:=\operatorname{det}\left(T^{*} X\right)
$$

any volume form $d V$ on $X$ induces a smooth metric $\|\cdot\|_{d V}$ on $K_{X}$, by locally setting

$$
\|d z\|_{d V}^{2}:=c_{n} d z \wedge d \bar{z} / d V
$$

where $d z:=d z_{1} \wedge \cdots \wedge d z_{n}$ is the local holomorphic trivialization of $K_{X}$ induced by a choice of local holomorphic coordinates and $c_{n} d z \wedge d \bar{z}$ is a short hand for the local Euclidean volume form $\frac{i}{2} d z_{1} \wedge d \bar{z}_{1} \wedge \cdots \wedge \frac{i}{2} d z_{n} \wedge d \bar{z}_{n}$. When $d V$ is the volume form of a given Kähler metric $\omega$ on $X$, i.e. $d V=\omega^{n} / n$ !, then its curvature form may be identified with minus the Ricci curvature of $\omega$, i.e.

$$
\operatorname{Ric} \omega=-d d^{c} \log \frac{d V}{c_{n} d z \wedge d \bar{z}} .
$$

By a slight abuse of notation we will also write Ric $(d V)$ for the right hand side in formula 4.4 .

4.1.5. Twisted Kähler-Einstein metrics. A Kähler metric $\omega_{\beta}$ is said to be a twisted KählerEinstein metric if it satisfies the twisted Kähler-Einstein equation

$$
\operatorname{Ric} \omega=-\beta \omega+\theta,
$$

where the form $\theta$ is called the twisting form. Since $\omega_{\beta}$ is Kähler the form $\eta$ is necessarily closed and $J$-invariant, i.e. of type $(1,1)$. The corresponding equation at the level of cohomology classes is

$$
[\omega]=\frac{1}{\beta}\left([\theta]+c_{1}\left(K_{X}\right)\right)
$$

Remark 4.2. There is no loss of generality if one assumes that that $|\beta|=1$ (by replacing $\omega$ with $|\beta| \omega)$, but allow general $\beta$ makes the connection to the statistical mechanical framework more apparent. Moreover, allowing $\beta$ to vary continuously is important for Aubin's method of continuity [3].

To a given pair $\left(d V, \omega_{0}\right)$ consisting of a volume form $d V$ and a Kähler form $\omega_{0}$ on $X$ we associate, for any parameter $\beta \in \mathbb{R}$, the twisting form

$$
\theta:=\beta \omega_{0}+\operatorname{Ric} d V
$$

This association is invariant under $\left(d V, \omega_{0}\right) \longrightarrow\left(e^{\beta u} d V, \omega_{0}+d d u\right)$ for any $u \in C^{\infty}(X)$.

The following lemma follows directly from the expression 4.4 for the Ricci curvature of a Kähler metric:

Lemma 4.3. Let $X$ be a compact complex manifold endowed with a Kähler form $\omega_{0}$ and volume form $d V$. Then a Kähler form $\omega_{\beta} \in\left[\omega_{0}\right]$ solves the corresponding twisted KählerEinstein equation 4.5 iff $\omega_{\beta}:=\omega_{0}+d d^{c} \varphi_{\beta}$ for a unique $\varphi_{\beta} \in \mathcal{H}\left(X, \omega_{0}\right)$ solving the PDE

$$
\omega_{\varphi}^{n}=e^{\beta \varphi} d V
$$

The Aubin-Yau theorem may now be formulated as follows: 
Theorem 4.4. (Aubin-Yau) [2, 81] Assume given a compact complex manifold $X$, endowed with a Kähler form $\omega_{0}$ and a volume form $d V$. Then the PDE 4.8 admits, for any positive number $\beta \in] 0, \infty\left[\right.$, a unique solution $\varphi_{\beta} \in \mathcal{H}\left(X, \omega_{0}\right)$. Equivalently, given a closed $(1,1)-$ form $\theta$ such that the cohomology class $[\theta]+c_{1}\left(K_{X}\right)$ is positive (i.e. contains a Kähler form) there exists a unique Kähler metric $\omega_{\beta}$ in $\left([\theta]+c_{1}\left(K_{X}\right)\right) / \beta$ solving the twisted Kähler-Einstein equation 4.5 .

Proof. We recall that the uniqueness follows from the maximum principle, which also yields a priori $C^{0}(X)$-estimates. As for the existence it is shown using a method of continuity, based on the Aubin-Yau Laplacian estimates.

Example 4.5. A complex manifold $X$ admits a Kähler-Einstein metric with negative Ricci curvature iff $K_{X}$ is positive (and the metric is unique). Indeed, if $K_{X}$ is positive then, by the very definition of positivity, we can take $\omega_{0}:=-\operatorname{Ric} d V$ for some volume form on $X$, ensuring that $\theta=0$ above, with $\beta=1$ (and the converse is trivial).

4.1.6. The Fano setting. Let $X$ be a Fano manifold, i.e. $-K_{X}>0$ and fix a volume form $d V$ on $X$ with the property that Ric $d V>0$. The "Fano setting" will refer to the special situation when the geometric data is of the form $\left(d V, \omega_{0}\right)$ with

$$
\omega_{0}:=\operatorname{Ric} d V
$$

For any given $\beta \neq 0$ the corresponding twisted Kähler-Einstein equation 4.5 is then of the form

$$
\operatorname{Ric} \omega_{\beta}=-\beta \omega_{\beta}+(1+\beta) \operatorname{Ric} d V
$$

For $\beta=-1$ this is precisely the Kähler-Einstein equation 4.5 for a metric with positive Ricci curvature, while for a general $\beta \in[-1,0$ [ this is Aubin's continuity equation with "timeparameter"

$$
\gamma:=-\beta \in] 0,1]
$$

The fixed volume form $d V$ corresponds to a metric $\|\cdot\|$ on $-K_{X}$, which is the dual of the metric induced by $d V$ on $K_{X}$ (formula 4.3). In other words,

$$
\left\|\frac{\partial}{\partial z_{1}} \wedge \cdots \wedge \frac{\partial}{\partial z_{n}}\right\|^{2}:=d V / c_{n} d z \wedge d \bar{z}
$$

Denoting by $\phi_{0}$ the local weight of the metric we can thus locally express

$$
d V=c_{n} e^{-\phi_{0}} d z \wedge d \bar{z}, \quad d d^{c} \phi_{0}=\operatorname{Ric} d V
$$

Accordingly, the complex Monge-Ampère equation which is equivalent to Aubin's equation (Lemma 4.3) may be locally expressed as

$$
\left(d d^{c} \phi_{\beta}\right)^{n}=c_{n} e^{-\left(\gamma \phi+(1-\gamma) \phi_{0}\right)} d z \wedge d \bar{z}
$$

where $\phi_{\beta}:=\phi_{0}+\varphi_{\beta}$.

Theorem 4.6. (Bando-Mabuchi [4]) For $\beta>-1$ the equation 4.9 admits at most one solution and for $\beta=-1$ a solution is uniquely determined modulo the action of the group Aut $(X)_{0}$. 
Proof. The proof is based on Aubin's method of continuity [3], deforming from $\beta=0$ to $\beta=-1$,using the uniqueness at $\beta=0$ of the Calabi equation and the uniqueness property for the linearized equations for $\beta>-1$, which follows from the Bochner-Kodaira-Nakano inequality (which also holds for $\beta=-1$ when the group Aut $(X)_{0}$ is trivial). An alternative proof is given in [26] which generalizes to the log Fano setting, as discussed in Section 4.1.7.

4.1.7. The log Fano setting. Let $X$ be a complex manifold and $\Delta$ a $\mathbb{Q}$-divisor on $X$, i.e. a formal sum

$$
\Delta=\sum_{i=1}^{m} w_{i} \Delta_{i}
$$

of irreducible subvarieties $\Delta_{i}$ of codimension one in $X$, with coefficients $w_{i} \in \mathbb{Q}$. The pair $(X, \Delta)$ is called a log pair 61 and $(X, \Delta)$ is called a log Fano manifold if the anti-canonical line bundle $L$ of the pair $(X, \Delta)$ is positive

$$
L:=-\left(K_{X}+\Delta\right)>0,
$$

where we have identified $\Delta$ with the $\mathbb{Q}$-line bundle $L_{\Delta}$ defined by $\Delta$, which admits a (multivalued) meromorphic section $s_{\Delta}$ with multiplicities $w_{i}$ along $\Delta_{i}$ (this means that $s_{\Delta}^{\otimes l}$ is a well-defined meromorphic section of the line-bundle $l L_{\Delta}$ for any sufficiently divisible positive integer $l$ ). We fix such a section $s_{\Delta}$ (which is uniquely determined up to a non-zero multiplicative constant).

Fix a metric $\|\cdot\|$ on $L$ with positive curvature form $\omega_{0}$. Using that $s_{\Delta}$ defines a canonical (multi-valued) trivialization of the line bundle $L_{\Delta}$ on $X-\Delta$ we then get a measure on $X$ defined as in the previous section on $X-\Delta$ and then extended by zero to all of $X$. In other words, locally on $X$ we can express

$$
\mu_{0}=c_{n} e^{-\phi_{0}+\phi_{\Delta}} d z \wedge d \bar{z}, \quad
$$

where $\phi_{0}$ is the weight of the metric $\|\cdot\|$ on $L$ and $\phi_{\Delta}$ is the weight of the singular metric on $L_{\Delta}$ induced by $s_{\Delta}$. This formula shows that, in the log smooth case, $\mu_{0}$ has an $L_{l o c}^{p}$-density for some $p>1$ iff all coefficients of $\Delta$ are in ] $-\infty, 1\left[\right.$. In general, if $\mu_{0}$ has a $L_{l o c}^{p}$-density for some $p>1$ then the log pair $(X, \Delta)$ is said to be (sub) klt (which, in algebraic terms means that the log canonical threshold of $(X, \Delta)$ is $>1$ [61, 19]). Moreover, formula 4.10 reveals that

$$
\text { Ric } \mu_{0}-[\Delta]=\omega_{0},
$$

where $[\Delta]$ denotes the current of integration defined by $\Delta$. The "log Fano setting" will refer to the situation when the geometric data is of the form $\left(\mu_{0}, \omega_{0}\right)$ as above. In this setting, $\varphi_{\beta} \in \mathcal{E}^{1}(X)$ satisfies

$$
\left(\omega_{0}+d d^{c} \varphi_{\beta}\right)^{n}=e^{\beta \varphi_{\beta}} \mu_{0}
$$

iff the $(1,1)$ current $\omega_{\beta}:=\omega_{0}+d d^{c} \varphi_{\beta}$ satisfies

$$
\operatorname{Ric} \omega_{\beta}-[\Delta]=-\beta \omega_{\beta}+(1+\beta) \operatorname{Ric} d V
$$

in a weak sense [19]. In particular, for $\beta=-1$ this is the log Kähler-Einstein equation 3.16 for the log pair $(X, \Delta)$. 
4.2. Pluripotential theory. Recall first that a function $\phi(z)$ in $\mathbb{C}^{n}$, taking values in $[-\infty, \infty[$ is said to be plurisubharmonic (psh, for short) if it is subharmonic along all complex lines. Equivalently, this means that $\phi$ can be written as a decreasing limit of smooth functions $\phi_{j}$ such that $d d^{c} \phi_{j}$ is strictly positive, i.e defines a Kähler form. For $\phi$ psh $d d^{c} \phi$ defines a positive $(1,1)$-current. Coming back to the global setting of a compact complex manifold $X$ endowed with a holomorphic line bundle $L$ a singular metric $\|\cdot\|$ om $L$ (taking values in $[0, \infty[$ ) is said to be $p s h$, i.e. $\phi \in \mathrm{PSH}(L)$, if the corresponding local functions $\phi$ (formula 4.1) are psh. The curvature of a psh metric on $L$ thus defines a global positive $(1,1)$-current on $X$. When $L$ is positive, i.e. admits a smooth reference metric $\|\cdot\|_{0}$ whose curvature form $\omega_{0}$ defines a Kähler form on $X$, we can identify $P S H(X, L)$ with the space $P S H\left(X, \omega_{0}\right)$ of all $\omega_{0}$-psh functions $\varphi$ on $X$, i.e.

$$
P S H(X, L) \longleftrightarrow P S H\left(X, \omega_{0}\right):=\left\{\varphi \in L^{1}(X): \omega_{\varphi} \geq 0\right\},
$$

where $\varphi$ is assumed to be a strongly usc function (in order to make the representation in $L^{1}(X)$ unique). By Demailly's global approximation result a function $\varphi$ is in $P S H\left(X, \omega_{0}\right)$ iff it can be written as a decreasing limit $\varphi_{j}$ in $\mathcal{H}\left(X, \omega_{0}\right)$.

Example 4.7. Any element $s_{k} \in H^{0}(X, k L)$ induces a singular psh metric $\phi$ on $L$ corresponding to $\varphi \in H^{0}(X, k L)$ defined by $\varphi(x):=k^{-1} \log \left\|s_{k}\right\|^{2}(x)$. Thus the curvature of $\phi$ is the current of integration along the subvariety defined by the zero-locus of $s$, including multiplicities.

4.2.1. The complex Monge-Ampère operator and the pluricomplex energy $E(\mu)$. For $\varphi \in \mathcal{H}\left(X, \omega_{0}\right)$ the complex Monge-Ampère measure $M A(\varphi)$ is the probability measure on $X$ defined by the normalized volume form of the Kähler form $\omega_{\varphi}$ :

$$
M A(\varphi):=\omega_{\varphi}^{n} / V \quad V:=\int_{X} \omega_{0}^{n}
$$

using exterior products. The map $\varphi \mapsto M A(\varphi)$, viewed as a one-form on the convex space $\mathcal{H}\left(X, \omega_{0}\right)$ is exact and thus admits a primitive, denoted by $\mathcal{E}$. In other words, $\mathcal{E}$ is the functional on $\mathcal{H}\left(X, \omega_{0}\right)$ defined by the property that

$$
d \mathcal{E}_{\mid \varphi}=M A(\varphi),
$$

in the sense that

$$
{\frac{d \mathcal{E}\left(\varphi_{0}+t\left(\varphi_{1}-\varphi_{0}\right)\right)}{d t}}_{\mid t=0}=\int_{X} M A(\varphi)\left(\varphi_{1}-\varphi_{0}\right)
$$

and the normalization condition $\mathcal{E}(0)=0$. Occasionally, we will write $\mathcal{E}_{\omega_{0}}$ to indicate the dependence of $\mathcal{E}$ on the normalization. Integrating the defining relation 4.12 along the affine line segment in $\mathcal{H}\left(X, \omega_{0}\right)$ between $\varphi_{0}:=0$ and $\varphi_{1}:=\varphi$ reveals that

$$
\mathcal{E}(\varphi)=\frac{1}{(n+1) V} \int_{X} \varphi \sum_{j=0}^{n} \omega_{\varphi}^{j} \wedge \omega_{\varphi}^{n-j}
$$

We will also denote by $\mathcal{E}_{\omega_{0}}$ the smallest upper semi-continuous extension of $\mathcal{E}_{\omega_{0}}$ to all of $P S H\left(X, \omega_{0}\right)$ and write

$$
\mathcal{E}^{1}(X):=\left\{\varphi \in P S H(X, \omega): \mathcal{E}_{\omega_{0}}(\varphi)>-\infty\right\},
$$


which is called the space of all functions on $X$ with finite energy. The differential property 4.12 still holds on the whole space $\mathcal{E}^{1}(X)$ if the Monge-Ampère measure $M A(\varphi)$ is defined in terms of non-polar products of positive currents (see [18]).

Now, following [18] the pluricomplex energy $E_{\omega_{0}}(\mu)$ of a probability measure $\mu$ is defined by

$$
\left.\left.E_{\omega_{0}}(\mu):=\sup _{\varphi \in \mathcal{E}^{1}(X)} \mathcal{E}_{\omega_{0}}(\varphi)-\langle\varphi, \mu\rangle \in\right]-\infty, \infty\right]
$$

on $\mathcal{P}(X)$. The functional $E_{\omega_{0}}$ thus defined is lsc on $\mathcal{P}(X)$ (since it is the the sup of lsc (affine) functionals, using that $\varphi$ is usc).

Example 4.8. In the classical case $n=1$, i.e. when $X$ is a Riemann surface,

$$
E_{\omega_{0}}(\mu)=\frac{1}{2} \int_{X} G_{0}(x, y) \mu \otimes \mu
$$

where $G_{0}(x, y)$ is the corresponding Green function, i.e. the symmetric lsc function in $L^{1}(X \times$ $X$ ) determined by $d d^{c} G_{0}(\cdot, y)=\omega_{0}-\delta_{y}$ and $\int_{X} G(x, y) \omega(x)=0$. In electrostatic terms this means that $E_{\omega_{0}}(\mu)$ is the classical Coulomb energy of a positive charge distribution $\mu$ on $X$ in the "neutralizing back-ground charge $\omega_{0}$ " (compare [6]).

As shown in [18] the Monge-Ampère operator yields a bijection

$$
\varphi \mapsto M A(\varphi)
$$

between the space $\mathcal{E}^{1}(X) / \mathbb{R}$ and the space of all probability measure of finite energy. The proof uses a direct variational approach where the potential $\varphi_{\mu}$ of a measure $\mu$ of finite energy, i.e. the solution,

$$
M A\left(\varphi_{\mu}\right)=\mu
$$

is obtained as the element $\mathcal{E}^{1}(X)$ realizing the sup defining $E_{\omega_{0}}(\mu)$ (formula 4.14). In particular,

$$
E_{\omega_{0}}(\mu)=\mathcal{E}_{\omega_{0}}\left(\varphi_{\mu}\right)-\left\langle\varphi_{\mu}, \mu\right\rangle
$$

Remark 4.9. In the case when $\mu$ is a volume form the existence of a smooth solution $\varphi_{\mu}$ was first shown by Yau [81] in the solution of the Calabi conjecture (the uniqueness of such solutions is due to Calabi).

Inverting the relation 4.12 reveals that the differential of $d E$ at a volume form in $\mathcal{P}(X)$ is given by

$$
d E_{\mid \mu}=-\varphi_{\mu} \in \mathcal{H}(X) / \mathbb{R}
$$

More generally, by [8, Prop 2.7] this formula holds on all of $\mathcal{P}(X)$ in the sense of sub-gradients. That is to say that for any two elements $\mu_{0}$ and $\mu_{1}$ in $\mathcal{P}(X)$ of finite energy

$$
E\left(\mu_{1}\right) \geq E\left(\mu_{0}\right)-\left\langle\varphi_{\mu}, \mu_{1}-\mu_{0}\right\rangle
$$


4.2.2. The psh-projection $P_{\theta}$ and the Legendre-Fenchel transform of E. The "psh-projection" is the operator $P_{\omega_{0}}$ from $C^{0}(X)$ to $P S H\left(X, \omega_{0}\right)$ defined as the following envelope:

$$
\left(P_{\omega_{0}} u\right)(x):=\sup _{\varphi \in P S H\left(X, \omega_{0}\right)}\{\varphi(x): \varphi \leq u\}
$$

Using the operator $P$ the pluricomplex energy $E_{\omega_{0}}$ may be realized as a Legendre-Fenchel transform (see the general definition 4.29 below):

Proposition 4.10. The pluricomplex energy $E_{\omega_{0}}$, extended by $\infty$ to the space $\mathcal{M}(X)$ of all signed measures on $X$, is the Legendre-Fenchel transform of the convex functional

$$
f(u):=-\mathcal{E}_{\omega_{0}}\left(P_{\omega_{0}}-u\right)
$$

Equivalently, this means that

$$
E_{\omega_{0}}(\mu)=\sup _{u \in C^{0}(X)} \mathcal{E}_{\omega_{0}}\left(P_{\omega_{0}} u\right)-\langle u, \mu\rangle .
$$

Proof. Since we have assumed that $L>0$ this follows readily from monotonicity arguments and the fact that any $\varphi \in P S H\left(X, \omega_{0}\right)$ is the decreasing limit of functions in $P S H\left(X, \omega_{0}\right) \cap C^{0}(X)$. But as shown in [6] the result, in fact, holds more generally for any big cohomology class.

We also recall the following differentiability result from [16], which plays a key rule in the variational approach to complex Monge-Ampère equations in [18]:

Theorem 4.11. The convex functional $f(u)$ on $C^{0}(X)$, defined by formula 4.20, is Gateaux differentiable and its differential at $u \in C^{0}(X)$ is given by

$$
(d f)(u)=M A\left(P_{\omega_{0}} u\right)
$$

4.3. Probability. We recall some basic notions of probability theory (covered by any standard textbook; see in particular [39] for an introduction to large deviation techniques). A probability space is a space $\Omega$ equipped with a probability measure $p$ and a collection $\mathcal{F}$ of $p$-measurable subsets $\mathcal{B} \subset \Omega$. For our purposes it will be enough to consider the case when $\Omega$ is a compact topological space and then we will always take $\mathcal{F}$ to be the collection of all Borel subsets of $\Omega$. In general, the space $\Omega$ is called the sample space and a measurable subset $\mathcal{B} \subset \Omega$ is called an event with

$$
\operatorname{Prob\mathcal {B}}:=p(\mathcal{B})
$$

interpreted as the probability of observing the event $\mathcal{B}$ when sampling from $(\Omega, p)$. A measurable function $Y: \Omega \rightarrow \mathcal{Y}$ on a probability space $(\Omega, p)$ is called a random element with values in $\mathcal{Y}$ and its law $\Gamma$ is the probability measure on $\mathcal{Y}$ defined by the push-forward measure

$$
\Gamma:=Y_{*} p
$$

(the law of $Y$ is often also called the distribution of $Y$ ). A sequence of random elements $Y_{N}: \Omega_{N} \rightarrow \mathcal{Y}$ of probability spaces $\left(\Omega_{N}, p_{N}\right)$, taking values in the same topological space $\mathcal{Y}$ are said to convergence in law towards a deterministic element $y$ in $\mathcal{Y}$ if the corresponding laws $\Gamma_{N}$ on $\mathcal{Y}$ converge to a Dirac mass at $y$ :

$$
\lim _{N \rightarrow \infty} \Gamma_{N}=\delta_{y}
$$


in the weak topology. In the present setting $\mathcal{Y}$ will always be a separable metric space with metric $d$ and then $Y_{N}$ converge in law towards the deterministic element $y$ iff $Y_{N}$ converge in probability towards $y$, i.e. for any fixed $\epsilon>0$

$$
\lim _{N \rightarrow \infty} p_{N}\left\{d\left(Y_{N}, y\right)>\epsilon\right\}=0 .
$$

Remark 4.12. The expectation of a random variable $Y$ it defined by

$$
\mathbb{E}(Y):=\int_{\Omega} Y p
$$

(aka the sample mean of $Y$ ) which defines an element in $\mathcal{Y}$. The statement that $Y_{N}$ converges in law towards a deterministic element $y$ equivalently means that $\mathbb{E}\left(Y_{N}\right) \rightarrow y$ and that $Y_{N}$ satisfies the (weak) law of large numbers, i.e. the probability that $Y_{N}$ deviates from its mean tends to zero, as $N \rightarrow \infty$.

A random point process with $N$ particles on a space $X$ is, by definition, a probability measure $\mu^{(N)}$ on the $N$-fold product $X^{N}$ (the $N$-particle space) which is symmetric, i.e. invariant under action of the group $S_{N}$ of permutations of the factors of $X^{N}$. The empirical measure of a given random point process is the following random measure

$$
\delta_{N}: X^{N} / S_{N} \rightarrow \mathcal{P}(X), \quad\left(x_{1}, \ldots, x_{N}\right) \mapsto \delta_{N}\left(x_{1}, \ldots, x_{N}\right):=\frac{1}{N} \sum_{i=1}^{N} \delta_{x_{i}}
$$

on $\left(X^{N}, \mu^{(N)}\right)$. The law of $\delta_{N}$ thus defines a probability measure on the space $\mathcal{P}(X)$, that we shall denote by $\Gamma_{N}$ :

$$
\Gamma_{N}:=\left(\delta_{N}\right)_{*} \mu^{(N)}
$$

Remark 4.13. The $j$-point correlation measure $\left(\mu^{(N)}\right)_{j}$ of the $N$-particle random point process is the probability measure on $X^{j}$ defined as the push-forward to $X^{j}$ of $\mu^{(N)}$ under projection $X^{N} \rightarrow X^{j}$, where $\left(x_{1}, \ldots, x_{N}\right) \mapsto\left(x_{i_{1}}, \ldots, x_{i_{j}}\right)$ for any choice of $j$ different indices $i_{1}, \ldots, i_{j}$. In particular, by symmetry,

$$
\mathbb{E}\left(\delta_{N}\right)=\left(\mu^{(N)}\right)_{1}
$$

Note that the exchangeable random variables $x_{1}, . ., x_{N}$ are independent iff $\mu^{(N)}$ is a tensor product measure, $\mu^{(N)}=\mu^{\otimes N}$, where $\mu$ is the law of any $x_{i}$.

4.3.1. Large Deviation Principles (LDP). The notion of a Large Deviation Principle (LDP), introduced by Varadhan, allows one to give a notion of exponential convergence in probability. The general definition of a Large Deviation Principle (LDP) for a general sequence of measures [39] is modeled on the classical Laplace method of "saddle point approximation" of integrals:

Definition 4.14. Let $\mathcal{Y}$ be a Polish space, i.e. a complete separable metric space. A sequence $\Gamma_{k}$ of measures on $\mathcal{Y}$ satisfies a large deviation principle with speed $r_{k}$ and rate function $I: \mathcal{Y} \rightarrow]-\infty, \infty]$ if

$$
\limsup _{k \rightarrow \infty} \frac{1}{r_{k}} \log \Gamma_{k}(\mathcal{F}) \leq-\inf _{\mu \in \mathcal{F}} I
$$


for any closed subset $\mathcal{F}$ of $\mathcal{Y}$ and

$$
\liminf _{k \rightarrow \infty} \frac{1}{r_{k}} \log \Gamma_{k}(\mathcal{G}) \geq-\inf _{\mu \in G} I(\mu)
$$

for any open subset $\mathcal{G}$ of $\mathcal{Y}$.

In the present setting $\Gamma_{N}$ will arise as the sequence of probability measures on $\mathcal{P}(X)$ defined as laws of the empirical measures $\delta_{N}$ (formula 4.22). Once the LDP has been established we can apply the following basic

Lemma 4.15. Let $Y_{N}$ be a sequence of random variables taking values in space $\mathcal{Y}$ which is a compact Polish space. If the laws $\Gamma_{N} \in \mathcal{P}(\mathcal{Y})$ of $Y_{N}$ satisfy a LDP at speed $N$ with a good rate functional $I$ which admits a unique minimizer $y_{*}$, then $Y_{N}$ converge in law towards $y_{*}$. More precisely,

$$
\operatorname{Prob}\left\{d\left(y_{N}, y_{*}\right) \geq \epsilon\right\} \leq C_{\epsilon} e^{-N / C_{\epsilon}}
$$

Proof. We recall the simple proof. First applying the LDP to $\mathcal{F}=\mathcal{G}=\mathcal{Y}$ gives $I\left(y_{*}\right)=0$. Since $y_{*}$ is the unique minimizer of $I$ it follows that $\inf I>0$ on the closed subset $\mathcal{F}_{\epsilon}$ of $\mathcal{Y}$ where $d\left(\cdot, y_{*}\right) \geq \epsilon$. Applying the upper bound 4.25 in the LDP to $\mathcal{F}_{\epsilon}$ thus concludes the proof of the desired deviation inequality.

In other words, the lemma says that risk that $Y_{N}$ deviates from $y_{*}$ is exponentially small. In order to establish the LDP we will have great use for the following alternative formulation of a LDP (see Theorems 4.1.11 and 4.1.18 in [39]):

Proposition 4.16. Let $\mathcal{Y}$ be a compact metric space and denote by $B_{\epsilon}(y)$ the ball of radius $\epsilon$ centered at $y \in \mathcal{Y}$. Then a sequence $\Gamma_{N}$ of probability measures on $\mathcal{P}$ satisfies a LDP with speed $r_{N}$ and a rate functional $I$ iff

$$
\lim _{\epsilon \rightarrow 0} \liminf _{N \rightarrow \infty} \frac{1}{r_{N}} \log \Gamma_{N}\left(B_{\epsilon}(y)\right)=-I(y)=\lim _{\epsilon \rightarrow 0} \limsup _{N \rightarrow \infty} \frac{1}{r_{N}} \log \Gamma_{N}\left(B_{\epsilon}(y)\right)
$$

In the present setting of a sequence of random point process with $N$ particles the previous proposition may be symbolically summarized as follows:

$$
\operatorname{Prob}\left(\frac{1}{N} \sum_{i=1}^{N} \delta_{x_{i}} \in B_{\epsilon}(\mu)\right) \sim e^{-r_{N} I(\mu)}
$$

when first $N \rightarrow \infty$ and then $\epsilon \rightarrow 0$.

We also recall the following classical result of Sanov, which is the standard example of a LDP for random point processes (describing the case when the particles $x_{1}, \ldots, x_{N}$ define independent variables with identical distribution $\left.\mu_{0}\right)$ :

Theorem 4.17. (Sanov) Let $X$ be a topological space and $\mu_{0}$ a finite measure on $X$. Then the laws $\Gamma_{N}$ of the empirical measures $\delta_{N}$ defined with respect to the product measure $\mu_{0}^{\otimes N}$ on $X^{N}$ satisfy an LDP with speed $N$ and rate functional the relative entropy $D_{\mu_{0}}$.

Proof. As explained in [39] this is a consequence of the general Gärtner-Ellis theorem (recalled in Section 6.2). From this point of view the rate functional $I$ arises as the Legendre-Fenchel transform of the functional $f(u)$ on $C(X)$ defined by $f(u):=\log \int e^{u} \mu_{0}$, which, by Jensen's inequality is given by $D_{\mu_{0}}$. 
Recall that the relative entropy $D_{\mu_{0}}$ (also called the Kullback-Leibler divergence or the information divergence in probability and information theory) is the functional on $\mathcal{P}(X)$ defined by

$$
D_{\mu_{0}}(\mu):=\int_{X} \log \frac{\mu}{\mu_{0}} \mu
$$

when $\mu$ has a density $\frac{\mu}{\mu_{0}}$ with respect to $\mu_{0}$ and otherwise $D_{\mu_{0}}(\mu):=\infty$. If $\mu_{0}$ is a probability measure, then $D_{\mu_{0}}(\mu) \geq 0$ and $D_{\mu_{0}}(\mu)=0$ iff $\mu=\mu_{0}$ (by Jensen's inequality).

Remark 4.18. The "physical entropy" is usually defined as

$$
S(\mu):=-D_{\mu_{0}}(\mu)
$$

In fact, Sanov's theorem can be seen as a mathematical justification of Boltzmann's original formula expressing the physical entropy $S$ as the logarithm of the number of microscopic states consistent with a given macroscopic state (using the characterization of a LDP in Prop 4.16).

4.4. Variational analysis. The notion of Gamma-convergence was introduced by de Georgi (see the book [30] for background on Gamma-convergence).

Definition 4.19. A sequence of functions $E_{N}$ on a topological space $\mathcal{M}$ is said to Gamma-converge to a function $E$ on $\mathcal{M}$ if

$$
\begin{array}{ccc}
\mu_{N} \rightarrow \mu \text { in } \mathcal{M} & \Longrightarrow & \liminf _{N \rightarrow \infty} E_{N}\left(\mu_{N}\right) \geq E(\mu) \\
\forall \mu & \exists \mu_{N} \rightarrow \mu \text { in } \mathcal{M}: & \lim _{N \rightarrow \infty} E_{N}\left(\mu_{N}\right)=E(\mu)
\end{array}
$$

Given $\mu$, a sequence $\mu_{N}$ as in the last point above is called a recovery sequence for $\mu$. The limiting functional $E$ is automatically lower semi-continuous on $\mathcal{M}$.

Example 4.20. In our complex-geometric setting we will first embed $X^{N} / S_{N}$ into $\mathcal{P}(X)$ using the empirical measure $\delta_{N}$ and define $E_{N}$ by formula 3.5, extended by $\infty$ to all of the space $\mathcal{M}(X)$ of signed measures on $X$. Then, as explained in Section 6, $E_{N}$ Gamma-converges towards the pluricomplex energy $E(\mu)$. This example illustrates that, in general, it is not the case that $\lim \sup _{N} E_{N}\left(\mu_{N}\right) \leq E(\mu)$. Indeed, for any sequence where two points, say $x_{1}$ and $x_{2}$, coincide, $E_{N}\left(\mu_{N}\right)=\infty$ ! For this reason Gamma-convergence is not preserved under multiplication by negative numbers.

Following [12] we will also have use for a weaker notion of convergence. Given a subset $\mathcal{S} \Subset \mathcal{X}$ we will say that $f_{j}$ Gamma-converge to $f$ relative to $\mathcal{S}$ if the existence of a recovery sequence in $\mathcal{X}$ is only demanded when $x \in \mathcal{S}$. The definition is made so that the following basic property holds:

Lemma 4.21. Let $\mathcal{X}$ be a compact topological space and assume that $f_{j}$ Gamma-converges to $f$ relative to a set $\mathcal{S}$ containing all minima of $f$. Then

$$
\lim _{j \rightarrow \infty} \inf _{\mathcal{X}} f_{j}=\inf _{\mathcal{X}} f
$$

Moreover, if $f$ admits a unique minimizer $x$, then any sequence $x_{i}$ of minimizers of $f_{j}$ converges towards $x$, as $j \rightarrow \infty$.

A general criterion for Gamma-convergence on $\mathcal{P}(X)$ can be obtained using duality in topological vector spaces, as next explained. Let $f$ be a function on a topological vector space 
$V$. The Legendre-Fenchel transform $f^{*}$ of $f$ is the following convex lower semi-continuous function $f^{*}$ on the topological dual $V^{*}$

$$
f^{*}(w):=\sup _{v \in V}\langle v, w\rangle-f(v)
$$

in terms of the canonical pairing between $V$ and $V^{*}$. In the present setting we will take $V=C^{0}(X)$ and $V^{*}=\mathcal{M}(X)$, the space of all signed Borel measures on a compact topological space $X$.

Proposition 4.22. Let $E_{N}$ be a sequence of functions on the space $\mathcal{P}(X)$ of probability measures on a compact space $X$ and assume that

$$
\lim _{N \rightarrow \infty} E_{N}^{*}(w)=f(w)
$$

for any $w \in C(X)$ and that $f$ defines a Gateaux differentiable function on $C(X)$. Then $E_{N}$ converges to $E:=f^{*}$ in the sense of Gamma-convergence on the space $\mathcal{P}(X)$, equipped with the weak topology.

See [10, Prop 4.4] for the proof.

\section{THE THERMODYNAMICAL FORMALISM}

In this section we recall the thermodynamical formalism introduced in 8 , and further developed in [19]. The main character is the free energy functional $F_{\beta}$ on $\mathcal{P}(X)$, which, from the probabilistic point of view, appears as the rate functional of the LDP for the random point processes. In particular, the lower semi-continuiuty properties of $F_{\beta}$ will play a crucial role in Section 7.

Remark 5.1. Regardless of the probabilistic motivations the thermodynamical formalism (in particular, the use of Legendre-Fenchel transforms) also sheds some new light on the standard functionals in Kähler geometry. This was, in particular, exploited in the singular setting of log Fano varieties in [19] and in the variational approach to the Yau-Tian-Donaldson conjecture in [20].

5.1. The case $\beta>0$. Let $X$ be a compact complex manifold. To the data $\left(d V, \omega_{0}\right)$ consisting of a volume form $d V$ and a Kähler form $\omega_{0}$ on $X$ and a parameter $\beta>0$ we attach the following free energy functional on $\mathcal{P}(X)$

$$
\left.\left.F_{\beta}(\mu):=\beta E_{\omega_{0}}(\mu)+D_{d V}(\mu) \in\right]-\infty, \infty\right]
$$

which is lsc and convex on $\mathcal{P}(X)$ (since both terms are).

Remark 5.2. In thermodynamics the free energy is usually defined as $E+\beta^{-1} D_{d V}$ (since it is the energy that is free to perform work after the "useless" thermal energy has been subtracted; compare Remark 4.18). This definition was also used in [8]. But here it will be convenient to use the rescaled version of the free energy above, since it facilitates the transition from positive to negative $\beta$.

Lemma 5.3. Assume that $\mu_{\beta}$ is a volume form. Then the differential of $F_{\beta}$ at $\mu$ is given by

$$
d F_{\beta}(\mu)=-\beta \varphi_{\mu}+\log \frac{\mu}{d V},
$$


i.e. if $\mu_{t}$ is an affine curve of volume forms in $\mathcal{P}(X)$, then

$$
\frac{d F_{\beta}\left(\mu_{t}\right)}{d t}=\left\langle-\beta \varphi_{\mu_{t}}+\log \frac{\mu_{t}}{d V}, \frac{d \mu_{t}}{d t}\right\rangle
$$

Proof. Since $d D(\mu)=\log \frac{\mu}{d V}$ this follows directly from formula 4.17.

As a consequence, if $\mu_{\beta}$ is a volume form which is a critical point of $F_{\beta}$ on $\mathcal{P}(X)$, then $\mu_{\beta}$ satisfies the mean field type equation

$$
-\beta \varphi_{\mu_{\beta}}+\log \frac{\mu_{\beta}}{d V}+\log Z=0
$$

for some positive constant $Z$, i.e.

$$
\mu_{\beta}=\frac{e^{\beta \varphi_{\mu}} d V}{Z}
$$

Equivalently, this means that the potential $\varphi_{\beta}$ of $\mu_{\beta}$ satisfies, after perhaps shifting $\varphi_{\mu}$ by a constant to ensure $Z=1$, the complex Monge-Ampère equation 4.8 .

$$
M A(\varphi)=e^{\beta \varphi_{\mu_{\beta}}} d V
$$

To a volume form $\mu_{\beta}$ minimizing $F_{\beta}$ on $\mathcal{P}(X)$ we attach the Kähler potential

$$
\varphi_{\beta}:=\frac{1}{\beta} \log \frac{\mu_{\beta}}{d V}
$$

and the Kähler form on $X$

$$
\omega_{\beta}:=\omega_{0}+d d^{c} \varphi_{\beta},
$$

which satisfies the twisted Kähler-Einstein equation 4.5 .

The following result was shown in [8] (see also [11] for singular generalizations):

Theorem 5.4. Assume given $\left(d V, \omega_{0}\right)$ as above and parameter $\beta>0$. Then the free energy functional $F_{\beta}(\mu)$ admits a unique minimizer $\mu_{\beta}$ on $\mathcal{P}(X)$. Moreover, $\mu_{\beta}$ is a volume form and the corresponding Kähler potential $\varphi_{\beta}$ and Kähler form $\omega_{\beta}$ satisfy the complex Monge-Ampère equation 4.8 and twisted twisted Kähler-Einstein equation 4.5, respectively.

Proof. By the Aubin-Yau Theorem 4.4 there exists a volume form $\mu_{\beta}$ solving the critical point equation 5.2. Indeed, we can take $\mu_{\beta}:=M A\left(\varphi_{\beta}\right)$, where $\varphi_{\beta}$ solves the complex MA-equation in Theorem 4.4. But $E$ is convex on $\mathcal{P}(X)$ (by its very definition as a sup of affine functions) and $D$ is strictly convex (by Jensen's inequality). Hence, it is enough to show that $\mu_{\beta}$ is a sub-gradient for $F_{\beta}$. But this follows from the sub-gradient relation 4.18 .

Remark 5.5. Instead of relying on the Aubin-Yau theorem the minimizer $\mu_{\beta}$ can be obtained by directly maximizing the functional $\mathcal{G}_{\beta}$, appearing in the following section, following the variational approach introduced in [18]. This alternative approach is important in the more general case of a big cohomology class, as well as in singular settings (see [11]). 
5.2. The case $\beta<0$. In the case when $\beta<0$ we define $F_{\beta}(\mu)$ by the same expression 5.1 , when $E_{\omega_{0}}(\mu)<\infty$ and otherwise we set $F_{\beta}(\mu)=\infty$. The definition is made so that we still have $\left.\left.F_{\mu}(\mu) \in\right]-\infty, \infty\right]$ with $F_{\mu}(\mu)<\infty$ iff both $E(\mu)<\infty$ and $D(\mu)<\infty$. Set

$$
\gamma=-\beta>0 \text {. }
$$

In order to the study the functional $F_{-\gamma}$ the following auxiliary "dual" functional on $C^{0}(X)$ turns out to be very useful:

$$
\mathcal{G}_{-\gamma}(u):=\mathcal{E}(P(u))-\frac{1}{\gamma} \log \int_{X} e^{-\gamma u} d V: C^{0}(X) \rightarrow \mathbb{R}
$$

Moreover, for $\varphi \in \mathcal{E}^{1}(X)$ we set

$$
\mathcal{G}_{-\gamma}(\varphi):=\mathcal{E}(\varphi)-\frac{1}{\gamma} \log \int_{X} e^{-\gamma \varphi} d V: \mathcal{E}^{1}(X) \rightarrow \mathbb{R}
$$

which is consistent with the previous notation since both functional coincide on the intersection of their domains. Note that the critical point equation for the functional $\mathcal{G}_{-\gamma}(\varphi)$ is precisely the Monge-Ampère equation [5.3. Moreover, as observed in [8] the functional $\mathcal{G}_{-\gamma}$ on $C^{0}(X)$ may be expressed in terms of termwise Legendre-Fenchel transforms of $F_{\beta}$, as exploited in the proof of the following result from [8]:

Lemma 5.6. The following holds:

$$
\inf _{\mathcal{P}(X)} \gamma F_{-\gamma}=-\sup _{C^{0}(X)} \mathcal{G}_{-\gamma}=-\sup _{\mathcal{E}^{1}(X)} \mathcal{G}_{-\gamma}
$$

Moreover, for any $\varphi \in \mathcal{E}^{1}(X)$

$$
\gamma F_{-\gamma}(M A(\varphi)) \geq-\mathcal{G}_{-\gamma}(\varphi)
$$

Proof. Let $f$ and $g$ be two lsc convex function on the dual $\mathcal{X}^{*}$ of a locally convex topological vector space $\mathcal{X}$. Then

$$
\inf _{\mathcal{X}}\left(g^{*}-f^{*}\right)=-\sup _{\mathcal{X}^{*}}(g-f)
$$

Indeed, this follows directly from the fact that the Legendre-Fenchel transform is decreasing and involutive on $\mathcal{X}$ (see Section 4.5.2 in [39]). Moreover, if $f$ is Gateaux differentiable, then, for any $u \in \mathcal{X}^{*}$,

$$
\left(g^{*}-f^{*}\right)(d f[u]) \geq-(g-f)(u),
$$

just using that $g^{*}(d f[u]) \geq\langle d f[u], u\rangle-g(u)$ and $f(u)=\langle d f[u], u\rangle-f(u)$. In particular, taking $\mathcal{X}$ to be the space $\mathcal{M}(X)$ of all signed measures on $X$ and setting $g(u):=\frac{1}{\gamma} \log \int_{X} e^{\gamma u} d V$ on $C^{0}(X)=\mathcal{M}(X)^{*}$ gives $g^{*}=\gamma D$ (compare the proof of Theorem 4.17). Moreover, by Prop 4.10, $E=f^{*}$ for $f(u):=-\mathcal{E}(P(-u)$ and hence applying formula 5.4 yields

$$
\inf _{\mathcal{P}(X)}(\gamma D-E)=-\sup _{C^{0}(X)}\left(\frac{1}{\gamma} \log \int_{X} e^{\gamma u} \mu+\mathcal{E}(P(-u))\right),
$$

which proves the first equality in the lemma (since the sup in the rhs above is invariant under $u \mapsto-u$ ). The second equality then follows from combining $P(u) \leq u$ with monotonicity and a simple approximation argument. Finally, the inequality [5.5, combined with the differentiability Theorem 4.11, yields the inequality in the lemma when $\varphi \in \mathcal{H}(X)$ and the general case then follows by approximation. 
The previous lemma is used in the proof of the following regularity result from [19]:

Theorem 5.7. (Regularity). Any minimizer $\mu_{\beta}$ of $F_{\beta}$ is a volume form and hence the corresponding Kähler form $\omega_{\beta}$ satisfies the twisted Kähler-Einstein equation 4.5.

Proof. If $\mu_{\beta}$ minimizes $F_{\beta}$ then, in particular, $E\left(\mu_{\beta}\right)<\infty$. Let $\varphi_{\beta} \in \mathcal{E}^{1}(X)$ be a potential for $\mu_{\beta}$, i.e. $M A\left(\varphi_{\beta}\right)=\mu$. It follows from the previous lemma that $\varphi_{\beta}$ maximizes the functional $G_{\beta}$ on $\mathcal{E}^{1}(X)$. But then it follows, as shown in [18, that $\phi_{\beta}$ satisfies the MA-equation 5.3 (using the differentiability of the functional $\mathcal{E} \circ P$ on $\mathcal{E}^{1}(X)+C^{0}(X)$, which follows from Theorem 4.11). Finally, as shown in the appendix of [19] (using Aubin-Yau type Laplacian estimates) any solution in $\mathcal{E}^{1}(X)$ is smooth, as desired.

It can be shown that $F_{\beta}$ is not bounded from below for $\beta$ sufficiently negative. In particular, it does not have a minimizer then. But we have the following

Theorem 5.8. Assume that the free energy functional $F_{\beta_{0}}$ is bounded from below for some $\beta_{0}<0$. Then for any $\beta>\beta_{0}$ the functional $F_{\beta}$ is lsc on $\mathcal{P}(X)$. In particular, it then admits a minimizer $\mu_{\beta} \in \mathcal{P}(X)$.

Proof. The lower semi-continuity follows from the results in [19], as we next recall. Take a sequence $\mu_{j} \rightarrow \mu_{\infty}$ in $\mathcal{P}(X)$. We may as well assume that $F_{\beta}\left(\mu_{j}\right) \leq C<\infty$ (otherwise there is nothing to prove). Since $F_{\beta_{0}} \geq C_{0}$ we get $D\left(\mu_{j}\right) \leq C_{1}$. But then it follows the energy-entropy compactness theorem in [19, Thm 2.17] that $\mu_{j} \rightarrow \mu_{\infty}$ in $\mathcal{P}(X)$ and $E\left(\mu_{j}\right) \rightarrow E\left(\mu_{\infty}\right)<\infty$. Since $D$ is lsc we deduce that $F_{\beta}\left(\mu_{\infty}\right) \leq \liminf { }_{j} F_{\beta}\left(\mu_{j}\right)$, as desired.

In general, a minimizer of $F_{\beta}$ need not be unique when $\beta<0$ and moreover there may be critical points which are not minimizers. However, the situation simplifies in the "Fano setting" (Section 4.1.6).

Theorem 5.9. Consider the "Fano setting" and fix $\beta \in[-1,0[$. In the case $\beta=-1$ we assume that the group Aut $(X)_{0}$ is trivial. Then the functional $F_{\beta}$ admits at most one minimizer. Moreover, the following is equivalent

(1) There exists a minimizer $F_{\beta}$ on $\mathcal{P}(X)$

(2) There exists $\epsilon>0$ such that the functional $F_{\beta-\epsilon}$ is bounded below on $\mathcal{P}(X)$, i.e. the following connectivity inequality holds for some constant $C_{\epsilon}$

$$
F_{\beta}(\mu) \geq \epsilon E_{\omega_{0}}-C_{\epsilon}
$$

(3) $F_{\beta}$ is lsc on $\mathcal{P}(X)$

Proof. Combining the regularity theorem with the uniqueness Theorem 4.6 for solutions to the twisted Kähler-Einstein-equation for $\omega_{\beta}$ shows that $F_{\beta}$ admits at most one minimizer. By the previous theorem all that remains is the implication " $1 \Longrightarrow 2$ ". We thus assume that there exists a minimizer $\mu_{\beta}$. By the regularity Theorem 5.7 this means that there exists a Kähler metric $\omega_{\beta}$ solving the twisted Kähler-Einstein-equation 4.5. Since the restriction of $F_{\beta}$ to the space of volume forms may be identified with the twisted Mabuchi functional (see Section 5.3) the connectivity inequality 5.6 restricted to the space of volume forms then follows from the corresponding coercivity inequality for the twisted Mabuchi functional on $\mathcal{H}\left(X, \omega_{0}\right)$, established in [76] Tian (using Aubin's method of continuity). Finally, if $\mu$ satisfies $F_{\beta}(\mu)<\infty$ we take a sequence of volume forms $\mu_{j}$ converging weakly towards $\mu$ such that $D\left(\mu_{j}\right) \rightarrow D(\mu)$. By the energy-entropy compactness theorem, as used in the proof of Theorem 5.8. $E\left(\mu_{j}\right) \rightarrow E(\mu)$ and hence the coercivity inequality holds on all of $\mathcal{P}(X)$. 
Remark 5.10. In the case when $\beta=-11$ and the group Aut $(X)_{0}$ it follows from [37 that the equivalence between the first two items still holds if the lower bound $E_{\omega_{0}}(\mu)$ is replaced by $\inf _{f} E_{\omega_{0}}\left(f_{*} \mu\right)$, where $f$ ranges over all element in Aut $(X)_{0}$.

We next observe that the lower-semi continuity of $F_{-1}$, in fact, forces the group Aut $(X)_{0}$ to be trivial, leading to the following equivalence:

Theorem 5.11. Let $X$ be a Fano manifold. Then the following is equivalent:

- $X$ admits a unique Kähler-Einstein metric

- The free energy functional $F_{-1}$ is lsc on $\mathcal{P}(X)$

Proof. By the previous theorem we just have to show that the second point implies the first point.We thus assume that $F_{-1}$ is lsc on $\mathcal{P}(X)$. By the previous theorem this implies that $X$ admits a Kähler-Einstein metric. Hence, by the uniqueness Theorem 4.6 we just have to show that the group Aut $(X)_{0}$ is non-trivial. Assume, to get a contradiction, that this is not the case. Then $X$ admits a non-trivial $\mathbb{C}^{*}$-action (since Aut $(X)_{0}$ is reductive when $X$ admits a Kähler-Einstein-metric). Denote by $\rho_{\tau}$ the corresponding family in Aut $(X)_{0}$, parameterized by $\tau \in \mathbb{C}^{*}$. Fix any volume form $d V_{1}$ on $X$ and set $d V_{\tau}:=\left(\rho_{\tau}\right)_{*} d V$. Note that $F\left(d V_{\tau}\right)$ is independent of $\tau$. Indeed, as recalled below $F(\mu)$ can be identified with the Mabuchi functional $\mathcal{M}$ and it is well-known that $\mathcal{M}$ is invariant under any $\mathbb{C}^{*}$-action, if there exists a Kähler-Einstein-metric. It follows from standard results that when $\tau \rightarrow 0$ the volume forms $d V_{\tau}$ converge weakly to a measure $\mu_{0} \in \mathcal{P}(X)$ supported on the fixed-point locus $Z$ in $X$ of the $\mathbb{C}^{*}$-action. In particular, $\mu_{0}$ is supported on a proper analytic subvariety of $X$. But, for any measure $\mu$ charging a pluripolar subset $E_{\omega_{0}}(\mu)=\infty$ [18. In particular, $F\left(\mu_{0}\right)=\infty$ and hence, $F$ cannot be lsc along the family $d V_{\tau}$. For completeness, we note that the converge of $d V_{t}$ towards $\mu_{0}$ used above, can be shown as follows. First note that $\rho_{0}(x):=\lim _{\tau \rightarrow 0} \rho_{\tau}(x)$ yields a well-defined continuous map $\rho_{0}$ from $X-Z$ into $Z$. Indeed, using that the $\mathbb{C}^{*}$ action lifts to $-K_{X}$ and that the Kodaira embedding 4.2 is $\mathbb{C}^{*}$-equivariant we can identify $X$ with a submanifold of $\mathbb{P}^{m}$ and the $\mathbb{C}^{*}$-action with the restriction to $X$ of a linear $\mathbb{C}^{*}$-action on $\mathbb{P}^{m}$. The existence of $\rho_{\infty}(x)$ then follows from the projective case, where the map in question is simply a rational projection from $\mathbb{P}^{m}$ onto a projective linear subspace. Hence, the limit $\mu_{0}$ is equal to the push-forward of $d V$ under the map $\rho_{\infty}$ (which is well-defined since $d V$ does not charge the locus $Z$ where $f_{\infty}$ is undetermined).

5.2.1. The log Fano setting. Now assume given a log Fano manifold $(X, \Delta)$, as defined in Section 4.1.7. Assume that $(X, \Delta)$ is (sub-)klt. We then consider the data $\left(\omega_{0}, \mu_{0}\right)$ consisting of a Kähler metric $\omega_{0} \in c_{1}\left(K_{X}+\Delta\right)$ and the induced measure $\mu_{0}$ on $X$ with singularities along $\Delta$, defined as in Section 3.2.4. In this setting we define the corresponding functional $F_{\beta}$ as in the Fano setting, but replacing the volume form $d V$ in formula 5.1 by the measure $\mu_{0}$. The following results are shown in [19].

Theorem 5.12. Consider a $\log$ Fano manifold $(X, \Delta)$. If the functional $F_{\beta}$ is bounded from below for some $\beta<-1$, then $F_{-1}$ admits a minimizer $\mu_{-1}$. Moreover, any minimizer $\mu_{-1}$ is given by a volume form on $X-\Delta$ and

$$
\omega_{-1}:=\omega_{0}-d d^{c} \log \frac{\mu_{-1}}{\mu_{0}}
$$


defines a Kähler metric on $X-\Delta$ which extends to a current in $-c_{1}\left(K_{(X, \Delta)}\right)$ solving the Kähler-Einstein-equation for $(X, \Delta)$. In the case when $\Delta$ is klt the minimizer is uniquely determined.

The uniqueness statement in the previous theorem follows from the generalization of the Bando-Mabuchi Theorem 4.6 to general singular log Fano varieties in [19, deduced from a variant of the convexity properties of the Ding functional along bounded geodesics in $P S H\left(X, \omega_{0}\right) \cap L^{\infty}(X)$ established in [26] (see also [32, the appendix of III]).

5.3. Relation to the standard functionals in Kähler geometry. First observe that it follows directly from formula 4.16, that if $\varphi) \in \mathcal{H}\left(X, \omega_{0}\right)$, then we can express

$$
E_{\omega_{0}}(M A(\varphi))=\left(I_{\omega_{0}}-J_{\omega_{0}}\right)(\varphi),
$$

where $I_{\omega_{0}}$ and $J_{\omega_{0}}$ are the standard energy type functionals in Kähler geometry defined by

$$
I_{\omega_{0}}(\varphi):=-\frac{1}{(n+1)} \int \varphi M A(\varphi)
$$

and

$$
I_{\omega_{0}}(\varphi):=\frac{1}{V} \int \varphi \omega_{0}^{n}-\mathcal{E}_{\omega_{0}}(\varphi)
$$

As is well-known all functionals $I_{\omega_{0}}, J_{\omega_{0}}$ and $I_{\omega_{0}}-J_{\omega_{0}}$ are non-negative (vanishing only for $\varphi=0$ ) and mutually compatible, with constants only depending on $n$ (see, for example, [18]).

In the "Fano setting" with $\beta=-1$ the functional

$$
\mathcal{D}(\varphi):=-\mathcal{G}_{-1}(\varphi):=-\mathcal{E}_{\omega_{0}}(\varphi)-\log \int_{X} e^{-\varphi} d V
$$

is the Ding functional introduced in [41. In the case of a general $\left(d V, \omega_{0}\right)$ the corresponding functional $-\mathcal{G}_{-1}(\varphi)$ can be viewed as a generalization of the Ding functional to the twisted setting.

Next, consider the functional $\mathcal{M}_{\beta}$ on $\mathcal{H}\left(X, \omega_{0}\right)$ defined by

$$
F_{\beta}(M A(\varphi))=: \mathcal{M}_{\beta}(\varphi)
$$

Lemma 5.13. Given the data $\left(d V, \omega_{0}\right)$ the differential of $\mathcal{M}_{\beta}$ at $\varphi \in \mathcal{H}\left(X, \omega_{0}\right)$ is given by

$$
d \mathcal{M}_{\beta}(\varphi)=-\frac{1}{V} n\left(\beta \omega_{\varphi}+\operatorname{Ric} \omega_{\varphi}-\theta\right) \wedge \omega_{\varphi}^{n-1}=:-\frac{n}{V}\left(\beta+n\left(R_{\omega_{\varphi}}-\operatorname{tr}_{\omega_{\varphi}} \theta\right)\right) \omega_{\varphi}^{n}
$$

where $R_{u}$ is the (normalized) scalar curvature of the Kähler metric $\omega_{u}$ and $\operatorname{tr}_{\omega_{u}} \theta$ is the trace of the twisting form $\theta$ with respect to $\omega_{\varphi}$.

Proof. Combining Lemma 5.3 with the chain rule and using that $d\left(M A\left(u_{t}\right)\right) / d t=V^{-1} n \omega_{u_{t}}^{n-1} \wedge$ $d d^{c}\left(d u_{t} / d t\right)$ gives

$$
d \mathcal{M}_{\beta}\left(\varphi_{t}\right) / d t=V^{-1} n\left\langle-\beta \varphi_{t}+\log \frac{M A\left(\varphi_{t}\right)}{d V}, \omega_{\varphi_{t}}^{n-1} \wedge d d^{c} \varphi_{t}\right\rangle .
$$

Integrating by parts to move $d d^{c}$ to the other side then concludes the proof, using that $R_{\omega}:=$ $\operatorname{tr}_{\omega} \operatorname{Ric} \omega:=n^{-1} \operatorname{Ric} \omega \wedge \omega^{n-1} / \omega^{n}$. 
For $\beta= \pm 1$ and $\theta=0$ we thus have that $\omega_{0} \in c_{1}\left(K_{X}\right)$

$$
d \mathcal{M}_{ \pm}(u)=-\frac{n}{V}\left( \pm \omega_{u}+\operatorname{Ric} \omega_{u}-\theta\right) \wedge \omega_{u}^{n-1}=:-\frac{n}{V}\left(\left( \pm 1+n R_{\omega_{u}}\right)\right) \omega_{u}^{n}
$$

which is the defining property of the Mabuchi functional on the space of Kähler potentials for $\pm c_{1}\left(K_{X}\right)$, introduced in [69] (which is thus only defined up to an additive constant). More generally, when $\beta= \pm 1$ and $\theta$ is a general closed one-form satisfying the cohomological equation 4.6 the formula 5.7 is the defining property of the corresponding twisted Mabuchi functional on the space of Kähler potentials for $\pm\left(c_{1}\left(K_{X}\right)+[\theta]\right)$.

5.3.1. Digression on constant scalar curvature. We make a brief digression to recall that the Mabuchi functional $\mathcal{M}_{\omega_{0}}$ is, in fact, defined for any Kähler class $\left[\omega_{0}\right]$ by the property

$$
d \mathcal{M}_{\omega_{0}}(u)=-\frac{1}{V} n\left(C_{0} \omega_{u}+\operatorname{Ric} \omega_{u}\right) \wedge \omega_{u}^{n-1},
$$

where $C_{0}$ is the cohomological constant ensuring that the right hand side above integrates to zero over $X$. This means that the critical points of $d \mathcal{M}_{\omega_{0}}$ in in $\mathcal{H}\left(X, \omega_{0}\right)$ are the Kähler potentials defining Kähler metrics with constant scalar curvature. Similarly, for any closed $(1,1)$-form $\theta$ there is a twisted Mabuchi functional on $\mathcal{H}\left(X, \omega_{0}\right)$ associated to the twisting form $\theta$ (obtained by replacing Ric $\omega_{u}$ with Ric $\omega_{u}-\theta$ and adjusting the cohomological constant $C_{0}$ accordingly). In this general setup there is variant of Theorem 5.9 saying that there exists a unique Kähler metric in $\left[\omega_{0}\right] \in H^{2}(X, \mathbb{R})$ iff the Mabuchi functional $\mathcal{M}$ is coercive on $\mathcal{H}\left(X, \omega_{0}\right)$. This is the content of Tian's properness conjecture, which was recently settled in [33] using a generalization of Aubin's continuity method. The "only if" direction was previously shown in [23], building on the general existence/properness principle in metric spaces established in [37]. Its application to Kähler geometry is based on the metric space realization of $\mathcal{E}^{1}(X)$ introduced in [36] together with the energy/entropy compactness theorem in [19] and the geodesic convexity of $\mathcal{M}$ in [16].

\section{The Large $N$-Limit in the CASE of Positive $\beta$}

In this section we will explain the key ideas in the proof in [10] of Theorem [3.3 (which implies Theorem 3.1 and also shows that Conjecture 3.8 is valid when $\beta>0$ ).

Let $(X, L)$ be a polarized manifold. Since $L$ is positive the sequence

$$
N_{k}:=\operatorname{dim} H^{0}(X, k L)
$$

tends to infinity, as $k \rightarrow \infty$. To the geometric data $(d V,\|\cdot\|)$ consisting of a volume form $d V$ on and a metric $\|\cdot\|$ on $L$ we attach, for any parameter $\beta \in] 0, \infty[$ the following sequence of probability measures $\mu_{\beta}^{\left(N_{k}\right)}$ on $X^{N_{k}}$ :

$$
\mu_{\beta}^{\left(N_{k}\right)}:=\frac{\left\|\operatorname{det} S^{(k)}\left(x_{1}, \ldots, x_{N_{k}}\right)\right\|^{2 \beta / k}}{Z_{N_{k}, \beta}} d V^{\otimes N_{k}},
$$

where $\operatorname{det} S^{(k)}$ is the holomorphic section of $(k L)^{\bigotimes N_{k}} \rightarrow X^{N_{k}}$ defined as a Slater determinant for $H^{0}(X, k L)$ :

$$
\left(\operatorname{det} S^{(k)}\right)\left(x_{1}, x_{2}, \ldots, x_{N}\right):=\operatorname{det}\left(s_{i}^{(k)}\left(x_{j}\right)\right),
$$

in terms of a given basis $s_{i}^{(k)}$ in $H^{0}(X, k L)$. A change of basis only has the effect of multiplying the section $\operatorname{det} S^{(k)}$ by a complex constant $c$ (the determinant of the change of bases matrix) 
and hence the probability measure $\mu^{\left(N_{k}\right)}$ is independent of the choice of basis. We will fix a basis which is orthonormal with respect to the scalar product on $H^{0}(X, k L)$ induced by the data $(d V,\|\cdot\|)$. As in Section 4.1 we denote by $\omega_{0}$ the curvature form of the fixed metric $\|\cdot\|$ on $L$.

Example 6.1. ("canonical case"). When $L=K_{X}$ and $\|\cdot\|$ is taken to be the metric on $K_{X}$ induced by the fixed volume form $d V$, the contributions from $\|\cdot\|$ and the volume form $d V$ cancel and then $\mu_{\beta}^{\left(N_{k}\right)}$ coincides with the canonical probability measure defined in Section 3.1 .

The following result from [10, Thm 5.7], was stated as Theorem 3.3 in Section 3 .

Theorem 6.2. Let $(X, L)$ be a polarized manifold and fix the geometric data $(d V,\|\cdot\|)$. Then, for any $\beta>0$, the the laws of the corresponding random measures $\delta_{N}$ on $\left(X^{N}, \mu_{\beta}^{(N)}\right)$ satisfy a Large Deviation Principle (LDP) with speed $N$ and rate functional $F_{\beta}(\mu)-C_{\beta}$, where

$$
F_{\beta}(\mu)=\beta E_{\omega_{0}}(\mu)+D_{d V}(\mu), C_{\beta}=\inf _{\mathcal{P}(X)} F_{\beta} .
$$

In particular,

$$
-\lim _{N \rightarrow \infty} N^{-1} \log Z_{N, \beta}=\inf _{\mathcal{P}(X)} F_{\beta}
$$

Since, by Theorem 5.4, there exists a unique minimizer $\mu_{\beta}$ of $F_{\beta}$ it follows (see Lemma 4.15) that $\delta_{N}$ converges in law towards the unique minimizer $\mu_{\beta}$. We recall that $\mu_{\beta}$ is a volume form and the Kähler metric

$$
\omega_{\beta}:=\omega_{0}+\frac{1}{\beta} d d^{c} \log \frac{\mu_{\beta}}{d V}
$$

is the unique solution to the twisted Kähler-Einstein equation

$$
\operatorname{Ric} \omega=-\beta \omega+\theta, \quad \theta:=\beta \omega_{0}+\operatorname{Ric} d V
$$

In particular, specializing the previous theorem to the "canonical case" in the previous example yields Theorem 3.1

6.1. The proof of Theorem 6.2. As mentioned in Section 3.1.1 the starting point of the proof of Theorem 3.3 is to rewrite $\mu^{\left(N_{k}\right)}$ as a Gibbs measure, at inverse temperature $\beta$,

$$
\mu_{\beta}^{\left(N_{k}\right)}=\frac{e^{-\beta N E^{(N)}}}{Z_{N, \beta}} d V^{\otimes N}, \quad E^{(N)}:=-\frac{1}{k N} \log \left\|\operatorname{det} S^{(k)}\left(x_{1}, \ldots, x_{N_{k}}\right)\right\|^{2}
$$

where, $E^{(N)}$ is called the energy per particle and the normalization constant $Z_{N, \beta}$ is called the partition function. To explain the idea of the proof first assume that that the following "Mean Field Approximation" holds in an appropriate sense

$$
E^{(N)}\left(x_{1}, \ldots x_{N}\right) \approx E\left(\frac{1}{N} \sum_{i=1}^{N} \delta_{x_{i}}\right), \quad N>>1
$$

for some functional $E$ on $\mathcal{P}(X)$. We are going to use the characterization 4.16 of a LDP. By definition, given $\mu \in \mathcal{P}(X)$ and $\epsilon>0$

$$
\operatorname{Prob}\left(\frac{1}{N} \sum_{i=1}^{N} \delta_{x_{i}} \in B_{\epsilon}(\mu)\right):=Z_{N, \beta}^{-1} \int_{\delta_{N}^{-1}\left(B_{\epsilon}(\mu)\right)} e^{-\beta N E^{(N)}} d V^{\otimes N}
$$


Hence, formally, as $N \rightarrow \infty$ and $\epsilon \rightarrow 0$, we can take out the factor $e^{-\beta N E^{(N)}}$ to get

$$
\int_{\delta_{N}^{-1}\left(B_{\epsilon}(\mu)\right)} e^{-\beta N E^{(N)}} d V^{\otimes N} \sim e^{-\beta N E(\mu)} \int_{\delta_{N}^{-1}\left(B_{\epsilon}(\mu)\right)} d V^{\otimes N}
$$

Applying the Sanov's LDP result 4.17 to the integral thus suggests that the non-normalized measures

$$
\left(\delta_{N}\right)_{*}\left(e^{-\beta H^{(N)}} d V^{\otimes N}\right)
$$

on $\mathcal{P}(X)$ satisfy a LDP with speed $N$ and rate functional

$$
F_{\beta}(\mu):=E(\mu)+\beta^{-1} D_{d V}(\mu) .
$$

Once this LDP has been established the asymptotics for $Z_{N}$ follow from the very definition of a LDP.

6.1.1. The two technical ingredients. In order to make this argument rigorous two issues need to be confronted. First, the nature of the convergence in the "Mean Field Approximation" 6.2 has to be specified. Secondly, appropriate conditions on $E^{(N)}$ need to be introduced, ensuring that the "taking out" argument 6.3 is justified. As for the first issue it is shown in [10, that, the approximation 6.2 holds in the sense of Gamma-convergence, with $E$ given by the pluricomplex energy $E_{\omega_{0}}$ (defined by formula 4.14). More precisely, using the embedding

$$
\delta_{N}: X^{N} / S_{N} \rightarrow \mathcal{P}(X)
$$

we can identify $E^{(N)}$ with a function on $\mathcal{P}(X)$, defined to be equal to $\infty$, on the complement of the image of $\delta_{N}$ in $\mathcal{P}(X)$. Under this identification it is shown in [10] that the following Gamma-convergence on $\mathcal{P}(X)$ holds:

$$
E^{(N)} \rightarrow E_{\omega_{0}}, \quad N \rightarrow \infty
$$

In fact, using the dual criterion in Prop 4.22, this follows directly from the Legendre-Fenchel formula for $E_{\omega_{0}}$ in Prop 4.10 and the differentiability Theorem 4.11, combined with the following convergence result for the weighted transfinite diameters of $X$ in [16]: given $u \in C^{0}(X)$

$$
\frac{1}{k N_{k}} \log \left\|\left(\operatorname{det} S^{(k)}\right) e^{-u / 2}\right\|_{L^{\infty}\left(X^{\left.N_{k}\right)}\right)}^{2} \rightarrow \mathcal{E}_{\omega_{0}}(P u), \quad k \rightarrow \infty,
$$

where we are using the same notation $u$ for the induced function $\sum_{i} u\left(x_{i}\right)$ on $X^{N_{k}}$. As for the "taking out" issue in formula 6.3 it is handled using the following key asymptotic sub-mean inequality in high dimensions established in [10]. There exist positive constants $C$ and $A_{\epsilon}$ such that for any $\boldsymbol{x}^{(N)} \in X^{N}$ and $\epsilon>0$

$$
e^{-\beta N E^{(N)}\left(\boldsymbol{x}^{(N)}\right)} \leq A_{\epsilon} e^{C N \epsilon} \frac{\int_{\delta_{N}^{-1}\left(B_{\epsilon}\left(\delta_{N}\left(\boldsymbol{x}^{(N)}\right)\right)\right.} e^{-\beta N E^{(N)}} d V^{\otimes N}}{\int_{\delta_{N}^{-1}\left(B_{\epsilon^{2}}\left(\delta_{N}\left(\boldsymbol{x}^{(N)}\right)\right)\right.} d V^{\otimes N}}
$$

when the metric $d$ on $\mathcal{P}(X)$, defining the weak topology, is taken to be the Wasserstein $L^{2}$-metric $d_{W^{2}}$. Combining the previous inequality with the Gamma-convergence 6.5] it is straightforward to conclude the proof of the LDP in Theorem 6.2 (see [10] and the exposition in [14]). 
6.1.2. The proofs of the technical ingredients. We briefly recall the proofs of the two technical ingredients discussed above. First, an important ingredient in the proof of the asymptotics 6.6] in [16] is the simple observation that the $L^{\infty}\left(X^{N}\right)$ norm in question has the same logarithmic asymptotics as the corresponding $L^{2}\left(X^{N}\right)$-norm. In turn, the $L^{2}\left(X^{N}\right)$-norm may, by expanding $\operatorname{det} S^{(k)}\left(x_{1}, \ldots x_{N}\right)$ as an alternating sum over the $N$ ! elements of $S_{N}$, be expressed as

$$
\left\|\operatorname{det} S^{(k)} e^{-k u}\right\|_{L^{2}\left(X^{\left.N_{k}\right)}\right.}^{2}=N_{k} \operatorname{det}_{i, j \leq N_{k}} A_{i j}[u],
$$

where $A_{i j}[u]$ is the $N \times N$ Gram matrix defined by the scalar products of the base elements $s_{i}^{(k)}$ in $H^{0}(X, k L)$ with respect to the scalar product induced by the volume form $d V$ and the metric $\|\cdot\| e^{-u / 2}$ on $L$. As shown in [16] the corresponding convergence towards $\mathcal{E}_{\omega_{0}}(P u)$ then follows from Bergman kernel asymptotics on $X$, using that the differential of the functional

$$
u \mapsto-\frac{1}{k N_{k}} \log \left\|\operatorname{det} S^{(k)} e^{-k u}\right\|_{L^{2}\left(X^{N_{k}}\right)}^{2}
$$

on $C(X)$ is represented by the probability measure on $X$ defined by the point-wise norm of the Bergman kernel on the diagonal, with respect to the metric $\|\cdot\| e^{-u / 2}$ on $L$.

Remark 6.3. Formula 6.8 allows one to view the functional in formula 6.9 as an analogue of Donaldson's $\mathcal{L}$-functional [43]. However, in contrast to the setting in [43], it is crucial that $u$ is allowed to be any continuous (or smooth) function and not only a function in $\mathcal{H}\left(X, \omega_{0}\right)$.

Finally, we recall the the starting point of the proof of the asymptotic submean inequality 6.7 in [10] is the well-known fact that the embedding $\delta_{N}$ of $X^{N} / S_{N}$ into the $L^{2}$-Wasserstein space $\left(\mathcal{P}(X), d_{W_{2}}\right)$ is an isometry when $X^{N} / S_{N}$ is endowed with the quotient space (orbifold) metric induced from the Riemannian metric $g_{N}$ on $X^{N}$, defined as $N^{-1}$ times the product Riemannian metric. The quasi-subharmonic property of $N E^{(N)}$ is equivalent to

$$
\Delta_{g_{N}} E^{(N)} \geq-\lambda
$$

on $X^{N}$. Moreover, the scaling of $g_{N}$ also ensures that the Ricci curvature of $g^{(N)}$ is bounded from below by a uniform constant times the dimension of $X^{N}$. The inequality 6.7 now follows from the general sub-mean inequality in [10, Thm 2.1] for Riemannian quotients (orbifolds) $Y:=M / G$ (which yields a distortion factor with sub-exponential growth in the dimension). We recall that the latter inequality is proved using geometric analysis on the orbifold $Y$, by generalizing an inequality of Li-Schoen in Riemannian geometry [67]. A key ingredient in the proof is the Cheng-Yau gradient estimate [34] for harmonic functions on a Riemannian manifold (or more generally orbifold) and the observation that the dependence on the dimension in the estimate is sub-linear.

6.2. A general LDP and the Gärtner-Ellis theorem. The same method of proof, in fact, yields the following general LDP:

Theorem 6.4. Let $E^{(N)}$ be a sequence of lower semi-continuous symmetric functions on $X^{N}$, where $X$ is a compact Riemannian manifold. Assume that

- The corresponding functions $E^{(N)}$ on $\mathcal{P}(X)$ converge to a functional $E$, in the sense of Gamma-convergence on $\mathcal{P}(X)$.

- $N E^{(N)}$ is uniformly quasi-superharmonic, i.e. $\Delta_{x_{1}} N E^{(N)}\left(x_{1}, x_{2}, \ldots x_{N}\right) \leq C$ on $X^{N}$ 
Then, for any sequence of positive numbers $\left.\left.\beta_{N} \rightarrow \beta \in\right] 0, \infty\right]$ the measures $\Gamma_{N}:=\left(\delta_{N}\right)_{*} e^{-\beta_{N} N E^{(N)}}$ on $\mathcal{P}(X)$ satisfy, as $N \rightarrow \infty$, a LDP with speed $\beta_{N} N$ and rate functional

$$
F_{\beta}(\mu)=E(\mu)+\frac{1}{\beta} D_{d V}(\mu)
$$

Moreover, assuming that the second point above holds, the first point may be replaced by the following assumption: there exists a sequence $\beta_{N} \rightarrow \infty$ such that for any $u \in C^{0}(X)$

$$
\mathcal{F}_{\beta_{N}}(u):=-\frac{1}{N \beta_{N}} \log Z_{N, \beta}[u], Z_{N, \beta}[u]:=\int_{X^{N}} e^{-\beta_{N}\left(N E^{(N)}+u\right)} d V^{\otimes N},
$$

converges, as $N \rightarrow \infty$, towards $\mathcal{F}(u)$ for some Gateaux differentiable functional $\mathcal{F}$ on $C^{0}(X)$. Then the Gamma-convergence in the first point above holds with $E$ defined as the LegendreFenchel transform of $f(u):=-\mathcal{F}(-u)$.

To see the connection between the last statement in the previous theorem and the present complex-geometric setup note that the functional in formula 6.9 can be expressed as $\mathcal{F}_{\beta_{N}}(u)$ for $\beta_{N_{k}}=k$. In this particular case, the corresponding random point processes is a determinantal point process (see [14, 47] for background on such processes and the relations to Fekete points and interpolation nodes).

As explained in [10], the previous theorem can be viewed as generalization of the GärtnerEllis theorem to $\beta \in] 0, \infty[$. The Gärtner-Ellis theorem (which is a generalization of Cramér's classical LDP theorem for independent random vectors) says, when applied to the laws of Gibbs measures, that the last assumption in Theorem 6.4 implies that LDP holds for $\beta=\infty$. However, extending the LDP to $\beta \in] 0, \infty[$ appears to require assumptions on the nature of $E^{(N)}$, such as the superharmonicity assumption in Theorem 6.4.

\section{TOWARDS THE CASE OF NEGATIVE $\beta$}

Now consider the "Fano setting" with $\beta \in[-1,0[$ (see Section 4.1.6) $)$. In order to extend the method of proof discussed in section to the case when $\beta<0$ it seems natural to expect that one would need to exploit that $\beta E^{(N}$ is uniformly quasi-plurisubharmonic. One small step in this direction is taken in the following

Lemma 7.1. There exists $\beta_{0}<0$ such that for any $\beta>\beta_{0}$ the following bound holds for a positive constant $C_{\beta}$ :

$$
N^{-1} \log Z_{N, \beta} \leq C_{\beta}
$$

Proof. Setting $\varphi^{(N)}:=-E^{(N)}$ on $X^{N}$, the functions on $X$ obtained by fixing all but one arguments in $\varphi^{(N)}$ are $\omega_{0}$-psh on $X$. By 6.6 there exists a uniform constant $C$ such that $\sup _{X^{N}} \varphi^{(N)} \leq C_{0}$. Moreover, as is well-known, there exists a positive number $\alpha$ such that for any $\gamma<\alpha$ there exists a constant $A_{\gamma}$ such that $\int_{X} e^{-\gamma \varphi} \leq A_{\gamma} e^{-\gamma \sup _{X} \varphi}$ for any $\varphi \in$ $\operatorname{PSH}\left(X, \omega_{0}\right)$. Indeed, the optimal such $\alpha$ is Tian's $\alpha$-invariant of $c_{1}\left(-K_{X}\right)$ aka as the global log canonical threshold of $X$ (see, for example, the appendix in [8], which applies to more general reference measure $\left.\mu_{0}\right)$. Hence, the lemma follows with $\beta_{0}=-\alpha$ and $C_{\beta}=\log \left(A_{\gamma}\right)+\gamma C_{0}$, by writing $Z_{N,-\gamma}$ as an iterated integral over the $N$ factors of $X^{N}$.

Here we will, however, propose a different variational route, based on Gibbs variational principle. As explained in [15] this approach is successful in the one-dimensional setting, but, in general, it hinges on a missing energy bound. 
It will be convenient to consider the setting of a general $\beta \in] 0,1]$. In order to get started we will make the following assumptions:

- The normalizing constant $Z_{N, \beta}$ is finite, i.e. the corresponding Gibbs measure $\mu_{\beta}^{(N)}$ is well-defined (formula 3.10).

- The free energy functional $F_{\beta}$ on $\mathcal{P}(X)$ has a unique minimizer $\mu_{\beta}$

The goal is then to prove that the random measure $\delta_{N}$ on $\left(X^{N}, \mu_{\beta}^{(N)}\right)$ converges in law towards the unique minimizer $\mu_{\beta}$ of $F_{\beta}$, i.e. that the convergence

$$
\Gamma_{N, \beta}:=\left(\delta_{N}\right)_{*} \mu_{\beta}^{(N)} \rightarrow \delta_{\mu_{\beta}}, \quad N \rightarrow \infty
$$

holds in the weak topology on

$$
\mathcal{X}:=\mathcal{P}(\mathcal{Y}), \quad \mathcal{Y}:=\mathcal{P}(X)
$$

We start by recalling Gibbs variational principle, which is a standard tool in Statistical Mechanics, involving the $N$-particle mean free energy functional $F_{\beta}^{(N)}$ on the space $\mathcal{P}\left(X^{N}\right)$ of all probability measures on $X^{N}$, defined by

$$
F_{\beta}^{(N)}\left(\mu_{N}\right):=\beta E^{(N)}\left(\mu_{N}\right)+D^{(N)}\left(\mu_{N}\right),
$$

where $E^{(N)}\left(\mu_{N}\right)$ denotes the $N$-particle mean free energy

$$
E^{(N)}\left(\mu_{N}\right):=\int_{X^{N}} E^{(N)} \mu_{N},
$$

and $D^{(N)}\left(\mu_{N}\right)$ denotes the $N$-particle mean entropy (relative to $d V^{\otimes N}$ )

$$
D^{(N)}\left(\mu_{N}\right):=D_{d V \otimes N}\left(\mu^{(N)}\right) / N
$$

Lemma 7.2. (Gibbs variational principle). Assume that $Z_{N, \beta}<\infty$. Then the Gibbs measure $\mu_{\beta}^{(N)}$ is the unique minimizer of the functional $F_{\beta}^{(N)}$ on $\mathcal{P}\left(X^{N}\right)$. Moreover,

$$
-\log Z_{N, \beta}=\inf _{\mathcal{P}\left(X^{N}\right)} F_{\beta}^{(N)}
$$

As a consequence, when $E^{(N)}$ is symmetric, i.e. $S_{N}$-invariant $\mu_{\beta}^{(N)}$ is the unique minimizer of $F_{\beta}^{(N)}$ on the space $\mathcal{P}\left(X^{N}\right)^{S_{N}}$ of all $S_{N}$-invariant probability measures on $X^{N}$ and

$$
-\log Z_{N, \beta}=\inf _{\mathcal{P}\left(X^{N}\right)^{S}} F_{\beta}^{(N)}
$$

Proof. This follows directly from rewriting $F_{\beta}^{(N)}\left(\mu_{N}\right)=-\log Z_{N, \beta}+D_{\mu_{\beta}^{(N)}}\left(\mu_{N}\right)$ and using that $D_{\nu}(\mu) \geq 0$ with equality iff $\mu=\nu$

Next, in order to study the limit $N \rightarrow \infty$ we embed all the spaces $\mathcal{P}\left(X^{N}\right)^{S^{N}}$ into the space $\mathcal{X}:$

$$
\left(\delta_{N}\right)_{*}: \mathcal{P}\left(X^{N}\right)^{S^{N}} \rightarrow \mathcal{X}
$$

We can then identify mean free energies $F^{(N)}$ with functionals on $\mathcal{X}$, extended by $\infty$ to all of $\mathcal{X}$. In particular, this means that we identity $\mathcal{P}(X)$ with its image in $\mathcal{X}$ under the embedding $\mu \mapsto \delta_{\mu}$. Consider now the following functional on $\mathcal{X}$ : 


$$
F_{\beta}(\Gamma)=\beta E(\Gamma)+D(\Gamma),
$$

defined when $E(\Gamma)<\infty$, where $E(\Gamma)$ and $D(\Gamma)$ are the affine functionals on $\mathcal{X}$ defined by

$$
E(\Gamma):=\int E(\mu) \Gamma, D(\Gamma):=\int D(\mu) \Gamma
$$

In the case when $E(\Gamma)=\infty$ we define $F_{\beta}(\Gamma):=\infty$. In order to prove the weak convergence 7.1 it is, in view of the previous lemma, enough to show the following conjectural convergence

$$
\lim _{N \rightarrow \infty} F_{\beta}^{(N)}=F, \text { on } \mathcal{X}
$$

in the sense of Gamma-convergence relative to some subset $\mathcal{S}$ of $\mathcal{X}$ containing the minima of $F$. Indeed, by Gibbs variational principle, if the previous convergence holds, then by Lemma 4.21 it is enough to show that the affine functional $F$ has the following property: it has a unique minimum and moreover the minimum is attained at $\delta_{\mu_{\beta}}$. But this follows from the following two results. First, we have the following elementary lemma:

Lemma 7.3. Suppose that $F(\mu)$ is lsc on $\mathcal{P}(X)$ and admits a unique minimizer $\mu_{*}$. Then $\delta_{\mu_{*}}$ is the unique minimizer of the affine functional $F(\Gamma)$ on $\mathcal{P}(X)$.

Proof. Since $F(\mu)$ is lsc we can write $F_{\beta}(\Gamma)=\int F_{\beta}(\mu) \Gamma$, which is lsc and affine on $\mathcal{X}$. Since $F_{\beta}(\Gamma)$ is affine we have

$$
\inf _{\mathcal{X}} F=\inf _{P(X)} F=F\left(\mu_{*}\right)
$$

After shifting $F(\mu)$ by a constant we may as well assume that $F\left(\mu_{*}\right)=0$. Take $\Gamma \neq \delta_{\mu_{*}}$. Then there exists a compact subset $K$ of $\mathcal{P}(X)$, not containing $\mu_{*}$ and such that $\Gamma(K)>0$. Moreover, since $F$ is lsc on $\mathcal{P}(X)$ we have $F(\mu) \geq \delta$ on $K$ for some $\delta>0$. But then $F(\Gamma) \geq$ $\delta \Gamma(K)>0=F\left(\mu_{*}\right)$, which concludes the proof.

Secondly we have the following

Lemma 7.4. The functional $F_{\beta}$ is lsc on $\mathcal{P}(X)$ and hence so is its affine extension to $\mathcal{X}$

Proof. Theorems 5.9 and 5.11 show that, in fact, $F_{\beta}$ is lsc iff it admits a unique minimizer, which we have assumed.

Remark 7.5. By general principles (see [12]) the strong LDP form of Conjecture 3.5, formulated in Conjecture 3.6 is, in fact, equivalent to having bona fide Gamma-convergence in 7.3 . But using the weaker relative notion may have some advantages, as discussed in Step 2 below.

We next take a first step towards proving the relative Gamma-convergence 7.3 .

Step 1: The existence of a recovery sequence. By Lemma 7.3 it is enough to prove the existence of a recovery sequence $\Gamma_{N}$ for any $\Gamma$ of the form $\Gamma=\delta_{\mu}$. To this end we set $\Gamma_{N}:=\left(\delta_{N}\right)_{*} \mu^{\otimes N}$ and first observe that the mean entropy is additive in the following sense:

$$
D^{(N)}\left(\mu^{\otimes N}\right)=D(\mu),
$$

for any given $\mu \in \mathcal{P}(X)$. Indeed, this follows directly from the additivity of log. Next observe that

$$
\liminf _{N \rightarrow \infty} E^{(N)}\left(\mu^{\otimes N}\right) \geq E(\mu)
$$


Indeed, fixing $u \in C^{0}(X)$ and rewriting

$$
E^{(N)}\left(\mu^{\otimes N}\right)=\int_{X^{N}}\left(E^{(N)}\left(x_{1}, \ldots, x_{N}\right)+N^{-1} \sum_{i=1}^{N} u\left(x_{i}\right)\right)-\int_{X} u \mu
$$

and estimating the integral over $X^{N}$ from below by its infimum, this follows directly from combining the asymptotics 6.6 with 4.10 . Hence, since $\beta<0$, this shows that

$$
\limsup _{N \rightarrow \infty} F_{\beta}^{(N)}\left(\Gamma_{N}\right) \leq F_{\beta}(\Gamma)
$$

as desired.

Towards the missing Step 2: the lower bound. We first recall the fundamental fact that mean entropy $D^{(N)}$ on $\mathcal{X}$ satisfies the lower bound in the definition of Gamma-convergence (as follows from basic sub-additive properties of the entropy; see [70] and [58, Thm 5.4]). In particular,

$$
D(\Gamma) \leq \liminf _{N \rightarrow \infty} D^{(N)}\left(\mu_{\beta}^{(N)}\right)<\infty
$$

Since $\beta<0$, it would thus be enough to show the following "upper bound property of the mean energy":

$$
\limsup _{N \rightarrow \infty} E^{(N)}\left(\mu_{\beta}^{(N)}\right) \leq E\left(\Gamma_{\beta}\right):=\int E(\mu) \Gamma_{\beta}(\mu)
$$

for any limit point $\Gamma_{\beta}$ in $\mathcal{X}$ of $\Gamma_{N, \beta}$. It should, however, be stressed that such bound can not hold for any sequence in $\mu^{(N)}$ in $\mathcal{P}\left(X^{N}\right)^{S_{N}}$, since $E(\mu)$ is not continuous on $\mathcal{P}(X)$. Note that, in general, $D(\Gamma)<\infty \Longrightarrow E(\Gamma)<\infty$, since $F_{\beta}(\Gamma)$ is bounded from below on $\mathcal{X}$ for some $\beta<-1$.

We summarize the output of the previous discussion, applied to the case $\beta=-1$, in the following

Theorem 7.6. Let $X$ be a Fano manifold and assume that $X$ is uniformly Gibbs stable. Then $X$ admits a unique Kähler-Einstein metric $\omega_{K E}$ and if the "upper bound property of the mean energy" 7.4 holds for the canonical sequence $\mu^{(N)}$, then the empirical measures $\delta_{N}$ of the canonical random point process on $X$ converge in law towards the normalized volume form $d V_{K E}$ of $\omega_{K E}$.

Proof. Set $\beta=-1$. If $X$ is uniformly Gibbs stable, then as shown in [53], $X$ is uniformly K-stable. Hence, by either [32] or [20] $X$ admits a unique Kähler-Einstein metric. Thus $F_{-1}$ admits a unique minimizer, $\mu_{-1}$, given by $d V_{K E}$. Moreover, by the uniform Gibbs stability $Z_{N, \beta}$ is finite for $N$ sufficiently large. Hence, the theorem follows from Step 1 and 2 above.

Remark 7.7. Note that if the "upper bound property of the mean energy" holds then the argument above shows that

$$
\lim _{N \rightarrow \infty} \inf _{\mathcal{P}\left(X^{N}\right)^{S_{N}}} F_{\beta}^{(N)}=\lim _{N \rightarrow \infty} \inf _{\mathcal{P}(X)^{\otimes N}} F_{\beta}^{(N)}
$$

i.e. asymptotically, as $N \rightarrow \infty$ the infimum of the mean free energy functional $F_{\beta}^{(N)}$ can be restricted to the subspace $\mathcal{P}(X)^{\otimes N} \Subset \mathcal{P}\left(X^{N}\right)^{S_{N}}$. 
7.1. Analyticity and absence of phase transitions. The proof of Theorem 7.6 reveals that in order to establish the convergence towards $d V_{K E}$ it is enough to show that

$$
-\lim _{N \rightarrow \infty} \frac{1}{N} \log Z_{N . \beta}=\inf _{\mathcal{P}(X)} F_{\beta}
$$

for $\beta=-1$. By Theorem 5.4 the converge does hold for $\beta \geq 0$ and the problem of extending the convergence to $\beta=-1$ can be connected to the theory of phase transitions in statistical mechanics. To see this first consider the following general setup. Let $H^{(N)}$ be a sequence of measurable functions ("Hamiltonians") on the measure spaces $\left(X_{N}, d V_{N}\right)$ such that the corresponding partition function

$$
Z_{N, \beta}:=\int_{X} e^{-\beta H^{(N)}} d V_{N}
$$

is finite for some $\beta>\beta_{0}$. Then $Z_{N, \beta}$ is real-analytic in $\beta$ on ] $-\beta_{0}, \infty[$ for any $N$ and strictly positive. However, in general $N^{-1} \log Z_{N, \beta}$ may converge to a function which is not realanalytic. This is often taken as the definition of a phase transition in statistical mechanics (see [71, Chapter 5]).

Example 7.8. The prime example of a phase transition is provided by the Curie-Weiss mean field model for magnetization in spin systems, where $N$ is the number of spins. If the sign convention for the corresponding Hamiltonians is taken so that $H^{(N)}$ is anti-ferromagnetic then the real-analyticity in question brakes down at a critical negative inverse temperature $\beta_{c}$ (in this case $\beta_{0}=-\infty$ ). This is precisely the inverse temperature for which the convergence of the empirical magnetization towards a deterministic limit fails (see the appendix in [14] for a comparison between the Curie-Weiss model and the present complex-geometric setup).

In the present Fano setting the partition function $Z_{N, \beta}$ is real-analytic in $\beta$, as long as $-\beta$ is strictly smaller than the stability threshold $\gamma_{N}$ (formula 3.13). In fact, according to well-known results of Atiyah and Bernstein-Gelfand the Archimedean zeta function $\beta \mapsto Z_{N, \beta}$ extends to a meromorphic function on $\mathbb{C}$ with a discrete set of rational poles located at $]-\infty, 0[\subset \mathbb{C}$ (see the book [56]). The next result shows that the convergence in Conjecture 3.5 holds in the absence of phase transitions down to the inverse temperature -1 :

Theorem 7.9. Assume that there exists $\beta_{0}<-1$ and a real-analytic function $f(\beta)$ on $]-$ $\beta_{0}, \infty[$ such that for any $\beta \in]-\beta_{0}, 0[$

$$
-\lim _{N \rightarrow \infty} \frac{\log Z_{N, \beta}}{N}=f(\beta) .
$$

Then $X$ admits a unique Kähler-Einstein-metric $\omega_{K E}$ and the empirical measures $\delta_{N}$ of the canonical random point process on $X$ converge in law towards the normalized volume form $d V_{K E}$ of $\omega_{K E}$.

Proof. Step 1: For $\beta \in\left[-1, \infty\left[\right.\right.$ the free energy functional $F_{\beta}$ admits a unique minimizer $\mu_{\beta}$ on $\mathcal{P}(X)$

To see this first recall that the argument in Step 1 of the proof of Theorem 7.6 shows that for $\beta \in\left[-1, \infty\left[\right.\right.$ we have $F_{\beta-\epsilon} \geq-C$ for some positive constants $C$ and $\epsilon$ (depending on $\beta$ ). Hence, by Theorems $5.8 F_{\beta}$ admits a unique minimizer $\mu_{\beta}$. In particular, there exists a unique Kähler-Einstein metric on $X$. 
Step 2: The function $F(\beta):=F\left(\mu_{\beta}\right)$ is real-analytic on $]-1, \infty[$ and continuous up to the boundary at $\beta=-1$

The continuity up to $\beta=-1$ was shown in 4. Now fix positive integers $p, l$ and consider the Banach (Hilbert) spaces defined by the Sobolev spaces $\mathcal{B}_{1}:=L^{p, l+2}(X)$ and $\mathcal{B}_{2}:=L^{p, l}(X)$. Take $p$ and $l$ sufficiently large so that $\mathcal{B}_{2} \subset C^{2}(X)$ (as ensured by the Sobolev inequality). Consider the map

$$
\left.g: \mathcal{B}_{1} \times\right]-\beta_{0}, \infty\left[\rightarrow \mathcal{B}_{2} \times\{0\}, \quad(u, \beta) \mapsto\left(\left(d d^{c} u\right)^{n} / d V-e^{\beta u}, \int_{X} \beta^{-1}\left(e^{\beta u}-1\right) d V\right)\right.
$$

(where $\beta^{-1}\left(e^{\beta t}-1\right)$ is defined to be equal to $t$ for $\beta=0$ ). The definition of $g$ is made so that $\varphi_{\beta}$ solves the MA-equation 6.6 iff $g\left(\varphi_{\beta}, \beta\right)=(0,0)$ and when $\beta=0$ the solution $\varphi_{\beta}$ is normalized so that $\int \varphi_{\beta} d V=0$. The map $g$ is a real-analytic map between Banach spaces in the sense of [77]. Indeed, $u \mapsto\left(d d^{c} u\right)^{n} / d V$ is continuous and multilinear (of order $2 n$ ) and the functions $e^{\beta t}$ and $\beta^{-1}\left(e^{\beta t}-1\right)$ are both real-analytic on $\mathbb{R} \times \mathbb{R}$. Next, note that the directional derivative $D_{u}(g, u)$ is surjective for $\beta \in[-1, \infty[$ and $u \in \mathcal{H}(X)$. Indeed, this is shown in the course of the proof of the openness property in Aubin's continuity path [2] (see also [4]). Hence, it follows from the real-analytic implicit function theorem in Banach spaces [77], that the curve $\varphi_{\beta}$ is real-analytic in $\mathcal{B}_{1}$. But then it follows from the explicit expression 4.13 for $\mathcal{E}(\varphi)$, which is a sum of multilinear terms in $\varphi$ that the function

$$
E(\beta):=E_{\omega_{0}}\left(\mu_{\beta}\right)=\mathcal{E}_{\omega_{0}}\left(\varphi_{\beta}\right)-\left\langle\varphi_{\beta}, M A\left(\varphi_{\beta}\right\rangle\right.
$$

is real-analytic on $]-1, \infty[$. The proof of Step 2 is now concluded by observing that $d F(\beta) / d \beta=$ $E(\beta)$. Indeed, this follows from the chain rule using that $\mu_{\beta}$ is the unique minimizer of $F_{\beta}$ on $\mathcal{P}(X)$.

Step 3: Conclusion of proof

By Theorem 6.2 $f(\beta)=F(\beta)$ for $\beta>0$ (note that the relation $f(0)=F(0)$ is trivial). Since $f$ and $F$ are both real-analytic on $]-1, \infty[$ and continuous as $\beta \rightarrow-1$ it follows that $f(\beta)=F(\beta)$ on all of $[-1, \infty[$. Hence, it follows from the proof of Theorem 7.6 that for any given $\beta \in\left[-1, \infty\left[\right.\right.$ the empirical measures $\delta_{N}$ on $\left(X^{N}, \mu_{\beta}^{(N)}\right)$ converge in law towards $\mu_{\beta}$. Indeed, since $f(\beta)=F\left(\mu_{\beta}\right)$ the assumed convergence can be used as a replacement for Step 2 in the proof of Theorem [7.6. Specializing to $\beta=-1$ thus concludes the proof.

Assuming the existence of a limiting function $f(\beta)$, one way of establishing the realanalyticity of $f$ is to show that the meromorphic extension of $Z_{N, \beta}$ to $\mathbb{C}$ is holomorphic and has no zeroes on some $N$-independent neighborhood of $[-1,0]$ in $\mathbb{C}$ (using Montel's convergence theorem of complex analysis). This is an approach that was pioneered by Lee-Yang for some statistical mechanical models, including spin models and lattice gases [80. Interestingly, in the present complex-geometric setup such a non-vanishing result holds in the setting of log Fano curves (in a slightly different setup), as shown in [15]. However, the general case is completely open.

\section{REFERENCES}

[1] M. F. Atiyah, M. F. Resolution of singularities and division of distributions. Comm.Pure Appl. Math,23,1970, 145-150.

[2] Aubin, T: Equations du type Monge-Amp'ere sur les vari ‘et 'es K” ahl'eriennes compactes. Bull. Sci. Math. (2) 102 (1978), no. 1, 63-95 
[3] Aubin, T: Réduction du cas positif de l'équation de Monge-Ampère sur les variétés kählériennes compactes à la démonstration d'une inégalité. J. Funct. Anal. 57 (1984), no. 2, 143-153

[4] S. Bando, T. Mabuchi: Uniqueness of Einstein K"ahler metrics modulo connected group actions. in Algebraic geometry, Sendai, 1985(T. Oda, Ed.), Adv. Stud. Pure Math.10, Kinokuniya,1987, 11-40

[5] Bayer, P.; Gu'ardia, J.: Hyperbolic uniformization of Fermat curves. Ramanujan J.12 (2006), no. 2, 207-223.

[6] Berman, R.J: Determinantal point processes and fermions on complex manifolds: Large deviations and Bosonization. Comm. in Math. Phys. Volume 327, Issue 1 (2014), Page 1-47

[7] Berman, R.J: Kahler-Einstein metrics emerging from free fermions and statistical mechanics. 22 pages, J. of High Energy Phys. (JHEP), Volume 2011, Issue 10 (2011)

[8] Berman, R.J: A thermodynamical formalism for Monge-Ampere equations, Moser-Trudinger inequalities and Kahler-Einstein metrics. Advances in Math. 1254. Volume: 248. 2013

[9] Berman, R.J: K-polystability of Q-Fano varieties admitting Kahler-Einstein metrics. Inventiones Math. March 2016, Volume 203, Issue 3, pp 973-1025

[10] Berman, R.J: Large deviations for Gibbs measures with singular Hamiltonians and emergence of KählerEinstein metrics. Communications in Mathematical Physics. Volume 354, Issue 3, pp 1133-1172 (2017)

[11] Berman, R.J: Kähler-Einstein metrics, canonical random point processes and birational geometry. Proceedings of Symposia in Pure Mathematics. Volume 97.1 : Algebraic Geometry Salt Lake City 2015 (Part 1). pp 29-74

[12] Berman, R.J: On large deviations for Gibbs measures, mean energy and Gamma convergence. Constructive Approximation. Volume 48, Issue 1 (2018) pp 3-30

[13] Berman, R.J; M. Onnheim: Propagation of chaos, Wasserstein gradient flows and toric Kahler-Einstein metrics. Anal. PDE Volume 11, Number 6 (2018), pp 1343-1380

[14] R.J. Berman: Statistical Mechanics of interpolation nodes, Pluripotential theory and Complex Geometry. Annales Polonici Mathematici - special Volume in honour of J.Siciak (to appear)

[15] Berman, R.J: Canonical metrics, point processes and vortices on algebraic curves. (In preparation)

[16] Berman, R.J.; Boucksom, S: Growth of balls of holomorphic sections and energy at equilibrium. Invent. Math. Vol. 181, Issue 2 (2010), p. 337

[17] Berman, R.J.; Boucksom, S; Witt Nyström, D: Fekete points and convergence towards equilibrium measures on complex manifolds. Acta Math. Vol. 207, Issue 1 (2011), 1-27

[18] Berman, R.J; Boucksom, S; Guedj,V; Zeriahi: A variational approach to complex Monge-Ampere equations. Publications math. de l'IHÉS (2012): 1-67, November 14, 2012

[19] R.J.Berman; Eyssidieu, P: S. Boucksom, V. Guedj, A. Zeriahi: Kähler-Einstein metrics and the KählerRicci flow on log Fano varieties. Journal fur die Reine und Angewandte Mathematik (published on-line 2016).

[20] Berman, R.J; Boucksom, S; Jonsson, M: A variational approach to the Yau-Tian-Donaldson conjecture. arXiv:1509.04561

[21] Berman, R.J; Boucksom, S; Jonsson, M: A Non-Archimedean approach to Gibbs stability (work in progress).

[22] R.J. Berman, B. Berndtsson, Convexity of the K-energy on the space of K“ahler metrics, J.Amer. Math. Soc. 30 (2017), no. 4, 1165-1196.

[23] R.J Berman, T Darvas, CH Lu: Regularity of weak minimizers of the K-energy and applications to properness and K-stabiliy. des Annales Scientifiques de l'École Normale Supérieure. 53, fasc. 2 (2020).

[24] Berman, R.J; Collins, T; Persson, D: The AdS/CFT correspondence and emergent Sasaki-Einstein metrics (in preparation).

[25] Berndtsson, B: Curvature of vector bundles associated to holomorphic fibrations. Annals of Math. Vol. 169 (2009), 531-560

[26] Berndtsson, B: A Brunn-Minkowski type inequality for Fano manifolds and some uniqueness theorems in Kähler geometry. Invent. Math. 200 (2015), no. 1, 149-200.

[27] Bernstein, I. N. and Gelfand, S. I. Meromorphy of the function $P^{\lambda}$. Funkcional. Anal.i Prilov zen.,3,1969,1,84-85.

[28] Blum, H.; Jonsson, M.; Thresholds, valuations, and K-stability. Adv. Math. 365 (2020),

[29] Boucksom, S; Hisamoto, T; Jonsson, M: Uniform K-stability, Duistermaat-Heckman measures and singularities of pairs. Ann. Inst. Fourier (Grenoble) 67 (2017), no. 2, 743-841 
[30] Braides, A: Gamma-convergence for beginners. Oxford University Press, 2002.

[31] Caglioti.E; Lions, P-L; Marchioro.C; Pulvirenti.M: A special class of stationary flows for two-dimensional Euler equations: a statistical mechanics description. Communications in Mathematical Physics (1992) Volume 143, Number 3, 501-525

[32] X.X. Chen, S. K. Donaldson and S. Sun.K“ahler-Einstein metrics on Fano manifolds, I-III. J. Amer.Math. Soc.28(2015), 183-197, 199-234, 235-278

[33] Chen, X, Cheng, J: On the constant scalar curvature Kähler metrics, existence results. https://arxiv.org/abs/1801.00656

[34] Cheng, S. Y.; Yau, S. T. Differential equations on Riemannian manifolds and their geometric applications. Comm. Pure Appl. Math. 28 (1975), no. 3, 333-354

[35] I. A. Cheltsov, Y. A. Rubinstein and K. Zhang. Basis log canonical thresholds, local intersection estimates, and asymptotically log del Pezzo surfaces. arXiv:1807.07135v2.

[36] T. Darvas, The Mabuchi Geometry of Finite Energy Classes, Adv. Math. 285 (2015)

[37] T. Darvas, Y. Rubinstein.Tian's properness conjectures and Finsler geometry of the space of Kahler metrics. J. Amer. Math. Soc.30(2017), 347-387

[38] Demailly, J: Complex Analytic and Differential Geometry. https://www-fourier.ujfgrenoble.fr / demailly/manuscripts/agbook.pdf

[39] Dembo, A; Zeitouni O: Large deviation techniques and applications. Jones and Bartlett Publ. 1993

[40] Dervan, R: Uniform stability of twisted constant scalar curvature Kähler metrics. Int. Math. Res. Not. IMRN 2016, no. 15, 4728-4783.

[41] Ding, W.: Remarks on the existence problem of positive Kähler-Einstein metrics. Math. Ann.463-472 (1988).

[42] Donaldson, S. K: Scalar curvature and projective embeddings. I. J. Differential Geom. 59 (2001), no. 3, $479-522$

[43] Donaldson, S. K. Scalar curvature and projective embeddings. II. Q. J. Math. 56 (2005), no. 3, 345-356.

[44] S. K. Donaldson,Some numerical results in complex differential geometry, Pure and ApplMath Quartly,5(2009), 571-618

[45] Donaldson, S.K: Stability of algebraic varieties and Kähler geometry. Algebraic geometry: Salt Lake City 2015, 199-221, Proc. Sympos. Pure Math., 97.1, Amer. Math. Soc., Providence, RI, 2018.

[46] Doran, C. F. :Picard-Fuchs uniformization: modularity of the mirror map and mirror-moonshine. The arithmetic and geometry of algebraic cycles (Banff, AB, 1998), 257-281, CRM Proc. Lecture Notes, 24, Amer. Math. Soc., Providence, RI, 2000.

[47] Dujardin, R: Theorie globale de pluripotentiel, equidistributions et processes ponctuels [d'après Berman, Boucksom, Witt Nyström,. . .]. Séminaire Bourbaki 2018-2019, no. 1153. http://www.bourbaki.ens.fr/TEXTES/Exp1153-Dujardin.pdf

[48] Elkies, N. D.:The Klein quartic in number theory. The eightfold way, 51-101, Math. Sci. Res. Inst. Publ., 35, Cambridge Univ. Press, Cambridge, 1999.

[49] Eyssidieux, P., Guedj, V., Zeriahi, A., Singular K“ahl er-Einstein metrics. J. Amer. Math. Soc. 22 (2009) no. 3, 607-639.

[50] Ferrari, F; Klevtsov, S; Zelditch, S: Random Kähler metrics. Nuclear Phys. B 869 (2013), no. 1, 89-110.

[51] Fujita, K; On Berman-Gibbs stability and K-stability of Q-Fano varieties,Compos. Math.152(2016), no. 2, 288-298

[52] Fujita, K: A valuative criterion for uniform K-stability of Q-Fano varieties. J. Reine Angew. Math. 751 (2019), 309-338.

[53] Fujita, Kento.J; Odaka, Y: On the K-stability of Fano varieties and anticanonical divisors. Tohoku Math. J. (2) 70 (2018), no. 4, 511-521.

[54] Guedj, V; Zeriahi, A: Degenerate Complex Monge-Ampere Equations. EMS Tracts in Mathematics (2017)

[55] Guenancia, H; Păun, M: Conic singularities metrics with prescribed Ricci curvature: general cone angles along normal crossing divisors. (English summary) J. Differential Geom. 103 (2016), no. 1, 15-57

[56] Igusa, J: An introduction to the theory of local zeta functions. AMS/IP Studies in Advanced Mathematics, 14. American Mathematical Society, Providence, RI; International Press, Cambridge, MA, 2000. xii +232 pp. ISBN: 0-8218-2015-X

[57] Jeffres, T; Mazzeo, R; Rubinstein, Y.A:; Kähler-Einstein metrics with edge singularities. Ann. of Math. (2) 183 (2016), no. 1, 95-176. 
[58] Hauray, M. and Mischler, S.On Kac's chaos and related problems. http://hal.archives-ouvertes.fr/hal-00682782

[59] Kiessling M.K.H.: Statistical mechanics of classical particles with logarithmic interactions. Comm. Pure Appl. Math. 46 (1993), 27-56.

[60] Kiessling M.K.H.: Statistical mechanics approach to some problems in conformal geometry. Phys. A 279 (2000), no. 1-4, 353-368.

[61] Kollar, J: Singularities of pairs. Algebraic geometry-Santa Cruz 1995, 221-287.

[62] M. Kontsevich and D. Zagier. Periods. Mathematics Unlimited - 2001 and Beyond771 - 809, SpringerVerlag, Berlin, 2001.

[63] A Kupiainen, R Rhodes, V Vargas: Integrability of Liouville theory: proof of the DOZZ Formula. Annals of Mathematics, 2020

[64] Li, C: K-semistability is equivariant volume minimization. Duke Math. J. 166, no. 16 (2017), 3147-3218

[65] C. Li. G-uniform stability and K“"ahler-Einstein metrics on Fano varieties. arXiv:1907.09399 3.

[66] C. Li, G. Tian and F. Wang. The uniform version of Yau-Tian-Donaldson conjecturefor singular Fano varieties. arXiv:1903.01215

[67] Li, P; Schoen, R: Lp and mean value properties of subharmonic functions on Riemannian manifolds. Acta Math. 153 (1984), no. 3-4, 279-301.

[68] Luo, F. and Tian, G.Liouville equation and spherical convex polytopes, Proc. Amer. Math. Soc. 116(1992), no. 4, 1119-1129

[69] Mabuchi, T: K-energy maps integrating Futaki invariants. Tohoku Math. J. (2) 38 (1986),no. 4, 575-593

[70] Robinson, D. W., and Ruelle, D. : Mean entropy of states in classical statistical mechanics.Comm.Math. Phys. 5(1967), 288-300

[71] D. Ruelle,Statistical mechanics. Rigorous results, Reprint of the 1989 edition (World Scientific Pub-lishing Co., Inc., River Edge, NJ; Imperial College Press, London, 1999).

[72] H. P. de Saint-Gervais: Uniformisation des surfaces de Riemann. Retour sur un théorème centenaire. ENS 'Editions, Lyon,2010. http://www.lcdpu.fr/livre/?GCOI=27000100107890 \&fa=complements

[73] Song, Y; Tian, G: Canonical measures and Kähler-Ricci flow. J. Amer. Math. Soc. 25 (2012), no. 2, 303-353.

[74] G. Székelyhidi, "Extremal metrics and K-stability (PhD thesis)",https://arxiv.org/abs/math/0611002, 2006.

[75] G. Székelyhidi, "Filtrations and test-configurations",Math. Ann.362(2015),no. 1-2, p. 451-484, With an appendix by Sébastien Boucksom

[76] Tian, G: K“*ahler-Einstein metrics with positive scalar curvature, Invent. Math.130(1997),no. 1, 1-37

[77] Tolands, J: http://www.dma.unina.it/hamiltonianPDE/mate/tolandCapri.pdf Buffoni, B; Toland, J: Analytic theory of global bifurcation. An introduction. Princeton Series in Applied Mathematics. Princeton University Press, Princeton, NJ, 2003.

[78] Tsuji,H: Canonical measures and the dynamical systems of Bergman kernels. Preprint arXiv:0805.1829. 2008

[79] Troyanov, M.Prescribing curvature on compact surfaces with conical singularities, Trans. Amer.Math. Soc. 324 (1991), no. 2, 793-821

[80] Yang, C. N.; Lee, T. D. (1952), Statistical Theory of Equations of State and Phase Transitions. I. Theory of Condensation. Physical Review, 87 (3): 404-409, Lee, T. D.; Yang, C. N. (1952). Statistical Theory of Equations of State and Phase Transitions. II. Lattice Gas and Ising Model", Physical Review, 87 (3): 410-419.

[81] Yau, S-T: On the Ricci curvature of a compact Kähler manifold and the complex Monge-Ampère equation. I. Comm. Pure Appl. Math. 31 (1978), no. 3, 339-411

[82] Yau, S. T.: Nonlinear analysis in geometry. Enseign. Math. (2) 33 (1987), no. 1-2, 109-158. 58-02

[83] S.-T. Yau, Open problems in Geometry, Proc. Symp. Pure Math.54(1993) 1-28

Robert J. Berman, Mathematical Sciences, Chalmers University of Technology and the University of Gothenburg, SE-412 96 Göteborg, Sweden

E-mail address: robertb@chalmers.se 